TRANSACTIONS OF THE

AMERICAN MATHEMATICAL SOCIETY

Volume 362, Number 1, January 2010, Pages 441-475

S 0002-9947(09)04791-6

Article electronically published on August 18, 2009

\title{
FLUCTUATIONS OF LÉVY PROCESSES AND SCATTERING THEORY
}

\author{
SONIA FOURATI
}

\begin{abstract}
Initial work by Spitzer was extended to show that the behavior of the bivariate processes $\left(X_{t}, \inf _{0 \leq s \leq t} X_{s}\right)$ or $\left(X_{t}, \sup _{0 \leq s \leq t} X_{s}\right)$, where $X$ is a Lévy process, can be entirely reconstructed on the basis of the Wiener-Hopf factorization of the Lévy exponent of $X$. This paper is meant to establish that a similar device can be used to investigate the trivariate Markov process $\left(X_{t}, \inf _{0 \leq s \leq t} X_{s}, \sup _{0 \leq s \leq t} X_{s}\right)$. This involves substituting (2,2)-matrices for the scalar functions involved in the Spitzer-type factorization. The computation of this matrix from the Lévy exponent of $X$ is a Riemann-Hilbert problem, which is the same as the one appearing in the inverse scattering problem.
\end{abstract}

\section{INTRODUCTION}

The relevance of the Wiener-Hopf theory to Lévy processes was discovered by Spitzer 22 and has given rise to a huge literature. Previous work on the subject was based on factoring $\phi(i u)+q$, where $\phi(i u)$ is the exponent of a Lévy process and $q$ is a nonnegative parameter, thus characterizing separately the law of the maximum $M$ and the law of the minimum $m$ of the process at an independent exponential time $\zeta$. In this paper, we substitute the matrix $\left(\begin{array}{cc}0 & -1 \\ 1 & \phi(i u)+q\end{array}\right)$ for $\phi(i u)+q$ and then use the Wiener-Hopf theory to factor it into two matrices. This turns out to provide enough information to reconstruct the joint distribution $(M, m)$. In fact, we obtain the distribution of the triple $(M, m, F)$, where $F$ is the value of the process at time $\zeta$.

This paper is organized as follows. In Section 2, we review the basic facts on Wiener-Hopf factorization. In Section 3, we introduce six functions which will play an important role in the rest of this work. In Section 4, we state the two main theorems. Theorem 4.1 provides a system of integral equations satisfied by the functions. Theorem 4.2 states that this system is equivalent to the Riemann-Hilbert problem mentioned above. In Section 5, we rephrase the previous results in terms of scattering theory and show that our six functions solve both a direct problem (Theorem 4.1) and an inverse problem (Theorem 4.2) in the sense of that theory. In Section 6 , we show, using appropriate path decompositions, that our six functions provide a unified treatment of a family of problems which includes the exit problem and the like. Technical work begins in Section 7. This section is devoted to a single

Received by the editors February 8, 2007 and, in revised form, March 28, 2008.

2000 Mathematics Subject Classification. Primary 60G51, 34L25; Secondary 60G52, 35Q15.

Key words and phrases. Lévy processes, fluctuation theory, Wiener-Hopf factorization, scattering theory, Riemann-Hilbert factorization.

(C)2009 American Mathematical Society Reverts to public domain 28 years from publication 
preliminary result to the effect that knowing that the amplitude doesn't exceed a given value, the pre and post minimum processes are independent. Sections 8 and 9 supply the proof of Theorem 4.1. In Section 8, we establish the first equation of the integral system of Theorem 4.1 Section 9 deals with the second group of equations. The key idea is to construct the solutions of the relevant equation from two Markov chains which are built from the successive minima and maxima of the process. Section 10 provides the proof of Theorem 4.2. There we develop Shabat's investigations (see 21]) into the Riemann-Hilbert problem to make them suitable for our purposes. Theorem 4.2 is interpreted probabilistically in Section 11. This is achieved by bringing out the relations between our six functions that appear in the factorization in the Riemann-Hilbert problem in terms of the usual WienerHopf factorizing of certain auxiliary Lévy processes. Finally, Section 12 is devoted to applying our results to stable processes and to Lévy processes without positive jumps. This allows us to extend the results initiated by Rogozin [19] such as those of Kyprianou [13] for the stable case. Lévy processes without positive jumps were first studied by Takács [24, followed by a large number of papers such as Emery [7, Koryluk, Suprun and Shurenkov [12, Suprun [23], Rogers [18, Bertoin [2], Avram, Kyprianou and Pistorius [1, Doney [6], Kyprianou and Palmowski [14, L. Nguyen-Ngoc and M. Yor [15], and Pistorius [16] and [17. Our version of these results provides a unified setting for these works.

\section{Some faCts on Wiener-Hopf FaCtorization}

Let $(\Omega, \mathcal{F})$ be the space of functions defined on $[0,+\infty[$ with values in $\mathbf{R} \cup\{\delta\}$, where $\delta$ is a cemetery point, and let $X$ denote the canonical process $X_{t}(\omega)=\omega(t)$. In this paper, $\mathbf{P}$ is the distribution on $(\Omega, \mathcal{F})$ of a Lévy process starting at 0 with Lévy exponent $\phi$. More precisely, $i u \mapsto \phi(i u)$ is the unique continuous function defined on the line $i \mathbf{R}$, such that $\phi(0)=0$ and which satisfies the following identity for all $i u \in i \mathbf{R}$ :

$$
e^{-t \phi(i u)}:=\mathbf{P}\left(e^{-i u X_{t}}\right) .
$$

We first remind the reader of the basic facts on the Wiener-Hopf factorization (see [3], chapter 6 , for more on the subject).

Let $S_{t}$ and $I_{t}$ be the past maximum and past minimum processes, namely:

$$
S_{t}:=\sup \left\{X_{s}, 0 \leq s \leq t\right\}, \quad I_{t}:=\inf \left\{X_{s}, 0 \leq s \leq t\right\} .
$$

We now introduce the local times at 0 of the reflected processes $X-S$ and $X-I$, $L$ and $\check{L}$, and the associated Wiener-Hopf factors $\psi_{q}(\lambda)$ and $\check{\psi}_{q}(\lambda)$. The definition of these objects involves the regularity of $[0,+\infty[$ or $]-\infty, 0]$ for the Lévy process $X$.

If $] 0,+\infty$ [ is regular (resp. ] $-\infty, 0\left[\right.$ ) i.e. $T^{0}=\inf \left\{t>0, X_{t}>0\right\}=0$ a.s. (resp. $T_{0}=\inf \left\{t>0, X_{t}<0\right\}=0$ a.s.), then 0 is a regular point for the Markov process $X-S($ resp. $X-I)$ and $L$ (resp. $\check{L}$ ) denotes any local time at 0 of this process. In this case, $t \mapsto L[0, t]$ (resp. $t \mapsto \check{L}[0, t]$ ) is an increasing continuous process, and we denote by $\tau_{t}$ (resp. $\check{\tau}_{t}$ ) its right continuous inverse and $H_{t}:=S_{\tau_{t}}$ (resp. $\left.\check{H}_{t}:=I_{\check{\tau}_{t}}\right)$. The pair $\left(\tau_{t}, H_{t}\right)$ (resp. $\left.\left(\check{\tau}_{t}, \check{H}_{t}\right)\right)$ is a bivariate Lévy process which has a finite lifetime if $\lim _{t \rightarrow+\infty} X_{t}=-\infty$ a.s. (resp. $\lim _{t \rightarrow+\infty} X_{t}=+\infty$ a.s.). Denote by $\psi_{q}(\lambda)$ (resp. $\left.\hat{\psi}_{q}(\lambda)\right)$ the Lévy exponent of $\left(\tau_{t}, H_{t}\right)$ (resp. $\left(\check{\tau}_{t}, \check{H}_{t}\right)$ ). More precisely, for every $q \in\left[0,+\infty\left[, \psi_{q}\right.\right.$ (resp. $\check{\psi}_{q}$ ) will be a continuous function defined on the 
half-plane $\{\lambda \mid \Re(\lambda) \geq 0\}$ (resp. $\{\lambda \mid \Re(\lambda) \leq 0\}$ ), $\psi_{q}(0)$ (resp. $\check{\psi}_{q}(0)$ ) is real, and $\psi_{q}(\lambda)$ (resp. $\check{\psi}_{q}(\lambda)$ ) satisfies the identity:

$$
e^{-t \psi_{q}(\lambda)}:=\mathbf{P}\left(e^{-\lambda H_{t}-q \tau_{t}} ; t<L_{\zeta}\right) \quad \Re(\lambda) \geq 0 ; q, t \in[0,+\infty[,
$$

respectively,

$$
e^{-t \check{\psi}_{q}(\lambda)}:=\mathbf{P}\left(e^{-\lambda \check{H}_{t}-q \check{\tau}_{t}} ; t<\check{L}_{\zeta}\right) \quad \Re(\lambda) \leq 0 ; q, t \in[0,+\infty[.
$$

If $\left[0,+\infty\right.$ [ is irregular (this means that the time $T^{0-}:=\inf \left\{t>0, X_{t} \geq 0\right\}$ is positive a.s.) (respectively if $]-\infty, 0]$ is irregular, $T_{0-}:=\inf \left\{t>0, X_{t} \leq 0\right\}$ is positive a.s.), then the set $\left\{t \mid S_{t}=X_{t}\right\}$ (resp. $\left\{t \mid X_{t}=I_{t}\right\}$ ) is a.s. discrete and the local time $L$ (respectively, $\check{L}$ ) is the random point measure

$$
L(d t):=\sum_{u \in[0, \zeta[} 1_{X_{u}=S_{u}} \delta_{u}(d t)
$$

respectively,

$$
\check{L}(d t):=\sum_{u \in[0, \zeta[} 1_{X_{u}=I_{u}} \delta_{u}(d t) .
$$

Denote by $\psi_{q}(\lambda)\left(\right.$ resp. $\left.\hat{\psi}_{q}(\lambda)\right)$ the functions

$$
\psi_{q}(\lambda):=1-\mathbf{P}\left(e^{-\lambda X_{T^{0-}}-q T^{0-}} ; T^{0-}<+\infty\right) \quad \Re(\lambda) \geq 0, q \in[0,+\infty[,
$$

respectively,

$$
\check{\psi}_{q}(\lambda):=1-\mathbf{P}\left(e^{-\lambda X_{T_{0-}-q T_{0-}}} ; T_{0-}<+\infty\right) \quad \Re(\lambda) \leq 0, q \in[0,+\infty[.
$$

Notice that, in this case, $\psi_{q}(\lambda)$ (resp. $\check{\psi}_{q}(\lambda)$ ) is the Lévy exponent of a bivariate compound Poisson process and that, according to proposition 4 of chapter 6 in $\underline{3}$, $X_{T^{0-}}>0$ a.s. (resp. $X_{T_{0-}}<0$ a.s.); thus the times $T^{0-}$ and $T^{0}:=\inf \left\{t, X_{t}>0\right\}$ are equal a.s. (resp. $T_{0-}=T_{0}:=\inf \left\{t ; X_{t}<0\right\}$ a.s.).

Finally, if none of these conditions is fulfilled, then $\mathbf{P}$ is the distribution of a compound Poisson process. In this case, for reasons which will appear later, it is necessary to use a dissymmetric definition of local times. We denote by $L$ the random measure

$$
L(d t):=1_{S_{t}=X_{t}} 1_{t \in[0, \zeta[} d t
$$

and $\breve{L}$ will be the random point measure

$$
\check{L}(d t):=\delta_{0}(d t)+\sum_{u \in] 0, \zeta[} 1_{I_{u^{-}}>I_{u}} 1_{X_{u}=I_{u}} \delta_{u}(d t) .
$$

As above, $\psi_{q}(\lambda)$ will denote the Lévy exponent of the bivariate Lévy process $\left(\tau_{t}, H_{t}\right)$, where $\left(\tau_{t}\right)$ is the inverse of the nondecreasing continuous function $t \mapsto L[0, t]$ and $H_{t}=S_{\tau_{t}}$; namely, $\psi_{q}(\lambda)$ is a continuous function defined on the half-plane $\{\lambda \mid$ $\Re(\lambda) \geq 0\}, \psi_{q}(0)$ is real, and $\psi_{q}(\lambda)$ satisfies the identity

$$
e^{-t \psi_{q}(\lambda)}:=\mathbf{P}\left(e^{-\lambda H_{t}-q \tau_{t}} ; t<L_{\zeta}\right) \quad \Re(\lambda) \geq 0 ; q, t \in[0,+\infty[.
$$

The function $\check{\psi}_{q}(\lambda)$ is the function defined by the following identity $\left(T_{0}:=\inf \left\{t, X_{t}\right.\right.$ $<0\}$ :

$$
\check{\psi}_{q}(\lambda):=1-\mathbf{P}\left(e^{-\lambda / l X_{T_{0}}-q T_{0}} ; T_{0}<\zeta\right) .
$$

Notice that again in this case, the function $\check{\psi}_{q}(\lambda)$ is the exponent of a bivariate compound Poisson process. 
Note that, in all cases,

$$
\frac{1}{\psi_{q}(\lambda)}=\mathbf{P}\left(\int_{[0, \zeta[} e^{-\lambda S_{t}-q t} L(d t)\right) \quad \Re(\lambda) \geq 0 ; q \in[0,+\infty[, q \Re(\lambda) \neq 0
$$

and

$$
\frac{1}{\breve{\psi}_{q}(\lambda)}=\mathbf{P}\left(\int_{[0, \zeta[} e^{-\lambda I_{t}-q t} \check{L}(d t)\right) \quad \Re(\lambda) \leq 0 ; q \in[0,+\infty[, q \Re(\lambda) \neq 0 .
$$

When $] 0,+\infty[$ (resp. ] $-\infty, 0[$ ) is regular, the local time $L$ (resp. $\check{L}$ ) is defined up to a multiplicative constant. In the sequel, this constant is chosen in such a way that Proposition 2.1 below holds. Notice that, for the compound Poisson process, this follows from the convention we have chosen.

Proposition 2.1. The pair $\left(\psi_{q}(\lambda), \check{\psi}_{q}(\lambda)\right)$ satisfies the following Wiener-Hopf identity:

$$
\check{\psi}_{q}(i u) \psi_{q}(i u)=\phi(i u)+q \quad i u \in i \mathbf{R} ; q \in[0,+\infty[.
$$

Note that the definitions above depart from ordinary use, as described, e.g., in Bertoin's book, in two respects. First, local times are defined here as random measures rather than nondecreasing functions. Second, by a Wiener-Hopf factor, we mean $\psi_{q}(\lambda)$ or $\check{\psi}_{q}(\lambda)$ instead of $\frac{\psi_{q}(0)}{\psi_{q}(i u)}$ or $\frac{\check{\psi}_{q}(0)}{\psi_{q}(i u)}$.

\section{The MAIn FUnCTiOns}

We now define the so-called excursion measures $N$ and $\check{N}$ associated to local times $\check{L}$ and $L$ by the compensation formula (see for example chapter 4 of [3]).

Proposition 3.1 (Compensation formula). There exists a unique measure on $(\Omega, \mathcal{F}), N$ (resp. $\check{N}$ ) such that

$$
\begin{gathered}
\mathbf{P}\left(\sum_{] g, d[\in \check{\mathcal{C}}} 1_{\left(I_{g}-X_{(g-s)}-\right)_{s \geq 0} \in d w_{1}} 1_{\left(I_{g}-X_{g+t}\right)_{0 \leq t<d-g} \in d w_{2}}\right) \\
=\mathbf{P}\left(\int_{[0,+\infty[} 1_{\left(I_{t}-X_{(t-s)}\right)_{s \geq 0} \in d w_{1}} \check{L}(d t)\right) N\left(d w_{2}\right),
\end{gathered}
$$

respectively,

$$
\begin{gathered}
\mathbf{P}\left(\sum_{] g, d[\in \mathcal{C}} 1_{\left(S_{g}-X_{(g-s)^{-}}\right)_{s \geq 0} \in d w_{1}} 1_{\left(S_{g}-X_{g+t}\right)_{0 \leq t<d-g} \in d w_{2}}\right) \\
=\mathbf{P}\left(\int_{[0,+\infty[} 1_{\left(S_{t}-X_{(t-s)^{-}}\right)_{s \geq 0} \in d w_{1}} L(d t)\right) \check{N}\left(d w_{2}\right),
\end{gathered}
$$

where $\check{\mathcal{C}}$ (resp. $\mathcal{C})$ is the set of connected components of the complement of the support of $\check{L}(d t)$ (resp. $L(d t))$.

Notice that if $[0,+\infty[$ (resp. $]-\infty, 0[$ ) is regular, then the state 0 is a regular point of the Markov process $X-S$ (resp. $X-I$ ) and $\check{N}$ (resp. $N$ ) is the usual excursion measure from 0 of this process. If $[0,+\infty[$ (resp. $]-\infty, 0[$ ) is irregular, then $\check{N}$ (resp. $N$ ) is the distribution under $\mathbf{P}$ of the canonical process $X$ killed at time $T^{0}$ (resp. $\left.T_{0}\right)$. 
We can now introduce the main functions of this paper. First, for each $x \in$ ] $0,+\infty[$ let

$$
\begin{aligned}
T^{x} & :=\inf \left\{t \mid X_{t}>x\right\}, \quad T_{x}:=\inf \left\{t \mid X_{t}<-x\right\}, \\
V_{x} & :=\inf \left\{t \mid X_{t}-S_{t}<-x\right\}, \quad V^{x}:=\inf \left\{t \mid X_{t}-I_{t}>x\right\} .
\end{aligned}
$$

Then, define the right continuous left limited functions of $x(x \in] 0,+\infty[)$ :

$$
\begin{aligned}
& A_{q}(x, \lambda):=\mathbf{P}\left(\int_{[0, \zeta[} 1_{S_{t}-I_{t} \leq x} e^{-\lambda S_{t}-q t} L(d t)\right) ; \quad \lambda \in \mathbf{C}, q \in[0,+\infty[, \\
& \check{A}_{q}(x, \lambda):=\mathbf{P}\left(\int_{[0, \zeta[} 1_{S_{t}-I_{t} \leq x} e^{-\lambda I_{t}-q t} \check{L}(d t)\right) ; \quad \lambda \in \mathbf{C}, q \in[0,+\infty[, \\
& C_{q}(x, \lambda):=N\left(e^{-\lambda X_{T^{x}}-q T^{x}} ; T^{x}<\zeta\right) ; \quad \Re(\lambda) \geq 0, q \in[0,+\infty[, \\
& \check{C}_{q}(x, \lambda):=\check{N}\left(e^{-\lambda X_{T_{x}}-q T_{x}} ; T_{x}<\zeta\right) ; \quad \Re(\lambda) \leq 0, q \in[0,+\infty[.
\end{aligned}
$$

If $\left[0,+\infty[\right.$ (resp. $]-\infty, 0[)$ is regular, it is easy to check that the process $\left(\left(\tau_{t} ; H_{t}\right)\right.$; $\left.0 \leq t<L_{V_{x}}\right)$ (resp. $\left.\left(\left(\check{\tau}_{t} ; \check{H}_{t}\right) ; 0 \leq t<\check{L}_{V^{x}}\right)\right)$ is a killed bivariate Lévy process, and we denote by $B_{q}(x, \lambda)$ (resp. $\check{B}_{q}(x, \lambda)$ ) its Lévy exponent; more precisely for every positive $x$ and every $q \in\left[0,+\infty\left[, B_{q}(x, \lambda)\right.\right.$ (resp. $\left.\breve{B}_{q}(x, \lambda)\right)$ is a continuous function defined on the half-plane $\{\lambda, \Re(\lambda) \geq 0\}$ (resp. $\{\lambda, \Re(\lambda) \geq 0\}$ ), $B_{q}(x, 0)$ (resp. $\left.\check{B}_{q}(x, 0)\right)$ is real and

$$
\begin{array}{ll}
e^{-t B_{q}(x, \lambda)}:=\mathbf{P}\left(e^{-\lambda S_{\tau_{t}}-q \tau_{t}} ; t<L_{V_{x}}\right) ; & \Re(\lambda) \geq 0, q, t \in[0,+\infty[, \\
e^{-t \check{B}_{q}(x, \lambda)}:=\mathbf{P}\left(e^{-\lambda I_{\check{\tau}_{t}}-q \check{\tau}_{t}} ; t<\check{L}_{V^{x}}\right) ; & \Re(\lambda) \leq 0, q, t \in[0,+\infty[.
\end{array}
$$

If $[0,+\infty[$ (resp. $]-\infty, 0[$ ) is irregular, then

$$
B_{q}(x, \lambda):=1-\mathbf{P}\left(e^{-\lambda S_{T^{0}}-q T^{0}} ; T^{0}<V_{x}\right) ; \quad \Re(\lambda) \geq 0, q \in[0,+\infty[,
$$

respectively,

$$
\check{B}_{q}(x, \lambda):=1-\mathbf{P}\left(e^{-\lambda I_{T_{0}}-q T_{0}} ; T_{0}<V^{x}\right) ; \quad \Re(\lambda) \leq 0, q \in[0,+\infty[.
$$

Notice that, in this case, $T^{0}<V_{x}$ if and only if $T^{0}<T_{x}$ (resp. $T_{0}<V^{x}$ if and only if $\left.T_{0}<T^{x}\right)$. Notice also that the function $(q, \lambda) \mapsto B_{q}(x, \lambda)\left(\operatorname{resp} .(q, \lambda) \mapsto \check{B}_{q}(x, \lambda)\right)$ is the Lévy exponent of a compound Poisson process.

In all cases, the following hold:

$$
\begin{array}{ll}
\frac{1}{B_{q}(x, \lambda)}=\mathbf{P}\left(\int_{\left[0, V_{x}[\right.} e^{-\lambda S_{t}-q t} L(d t)\right) ; & \Re(\lambda) \geq 0, q \in[0,+\infty[, \\
\frac{1}{\check{B}_{q}(x, \lambda)}=\mathbf{P}\left(\int_{\left[0, V^{x}[\right.} e^{-\lambda I_{t}-q t} \check{L}(d t)\right) ; & \Re(\lambda) \leq 0, q \in[0,+\infty[.
\end{array}
$$

We now define the following functions:

$$
\begin{aligned}
& H_{q}(x):=A_{q}(x, 0)=\mathbf{P}\left(\int_{[0, \zeta[} 1_{S_{t}-I_{t} \leq x} e^{-q t} L(d t)\right), \\
& \check{H}_{q}(x):=\check{A}_{q}(x, 0)=\mathbf{P}\left(\int_{[0, \zeta[} 1_{S_{t}-I_{t} \leq x} e^{-q t} \check{L}(d t)\right) .
\end{aligned}
$$


We will denote by $H_{q}(d x)$ and $\check{H}_{q}(d x)$ the Stieltjes measures associated to these nondecreasing functions. Since $L[0, \varepsilon]$ and $\check{L}[0, \varepsilon]$ are positive for every $\varepsilon>0, H_{q}(x)$ and $\check{H}_{q}(x)$ do not vanish for any positive $x$. Furthermore,

$$
H_{q}(x) \leq \mathbf{P}\left(\int_{[0, \zeta[} 1_{S_{t} \leq x} e^{-q t} L(d t)\right) \quad \text { and } \quad \check{H}_{q}(x) \leq \mathbf{P}\left(\int_{[0, \zeta[} 1_{-I_{t} \leq x} e^{-q t} \breve{L}(d t)\right) .
$$

For $q=0$, the right-hand side of the first (resp. second) inequality is the so-called renewal function of the subordinator with Lévy exponent $\psi_{0}(\lambda)\left(\right.$ resp. $\check{\psi}_{0}(-\lambda)$ ) (see [3] chapter 3). Therefore it is finite and $H_{q}(x)$ and $\check{H}_{q}(x)$ are finite too. Let us mention that the following inequalities have been proved in 8 :

$$
\mathbf{P}\left(\int_{[0, \zeta[} 1_{S_{t} \leq x} e^{-q t} L(d t)\right) \leq 4 H_{q}(x) \quad \text { and } \quad \mathbf{P}\left(\int_{[0, \zeta[} 1_{-I_{t} \leq x} e^{-q t} \check{L}(d t)\right) \leq 4 \check{H}_{q}(x) .
$$

\section{The MAIN RESUlts}

We now present the main results of this paper. These have been announced in [10.

Theorem 4.1. 1) For every $q \in[0,+\infty[$ and every complex number $\lambda$, the functions $A_{q}$ and $\check{A}_{q}$ satisfy the integral equations

$$
\begin{aligned}
A_{q}\left(x^{-}, \lambda\right) & =H_{q}(0)+\int_{] 0, x[} e^{-\lambda y} \check{A}_{q}(y, \lambda) \frac{H_{q}(d y)}{\check{H}_{q}(y)}, \\
\check{A}_{q}(x, \lambda) & =\check{H}_{q}(0)+\int_{] 0, x]} e^{\lambda y} A_{q}\left(y^{-}, \lambda\right) \frac{\check{H}_{q}(d y)}{H_{q}\left(y^{-}\right)} .
\end{aligned}
$$

2) For every $q \in[0,+\infty[$ and every complex number $\lambda$ with $\Re(\lambda)>0$ (and $\Re(\lambda)=0$ if $q>0$ or $\left.\lim X_{t}=-\infty\right)$ the functions $C_{q}$ and $B_{q}$ satisfy the integral equations

$$
\begin{aligned}
C_{q}\left(x^{-}, \lambda\right) & =\int_{[x,+\infty[} e^{-\lambda y} B_{q}(y, \lambda) \frac{H_{q}(d y)}{\check{H}_{q}(y)} \\
B_{q}(x, \lambda) & =\psi_{q}(\lambda)+\int_{] x,+\infty[} e^{\lambda y} C_{q}\left(y^{-}, \lambda\right) \frac{\check{H}_{q}(d y)}{H_{q}\left(y^{-}\right)} .
\end{aligned}
$$

Moreover,

$$
A_{q}\left(x^{-}, \lambda\right) B_{q}(x, \lambda)+\check{A}_{q}(x, \lambda) C_{q}\left(x^{-}, \lambda\right)=1 .
$$

3) For every $q \in[0,+\infty[$ and every complex number $\lambda$ with $\Re(\lambda)<0$ (and $\Re(\lambda)=0$ if $q>0$ or $\lim X_{t}=+\infty$ ) the functions $\check{C}_{q}$ and $\check{B}_{q}$ satisfy the integral equations

$$
\begin{aligned}
\check{C}_{q}(x, \lambda) & =\int_{] x,+\infty[} e^{\lambda y} \check{B}_{q}\left(y^{-}, \lambda\right) \frac{\check{H}_{q}(d y)}{H_{q}\left(y^{-}\right)} \\
\check{B}_{q}\left(x^{-}, \lambda\right) & =\check{\psi}_{q}(\lambda)+\int_{[x,+\infty[} e^{-\lambda y} \check{C}_{q}(y, \lambda) \frac{H_{q}(d y)}{\check{H}_{q}(y)} .
\end{aligned}
$$

Moreover,

$$
\check{A}_{q}(x, \lambda) \check{B}_{q}\left(x^{-}, \lambda\right)+A_{q}\left(x^{-}, \lambda\right) \check{C}_{q}(x, \lambda)=1 .
$$


For all $x \in] 0,+\infty[$ and all $q \in[0,+\infty[$ define

$$
\begin{aligned}
M_{q}(x, \lambda) & :=\left(\begin{array}{cc}
A_{q}(x, \lambda) & -C_{q}(x, \lambda) \\
\check{A}_{q}(x, \lambda) & B_{q}(x, \lambda)
\end{array}\right) \text { if } \quad \Re(\lambda)>0, \\
M_{q}(x, \lambda): & =\left(\begin{array}{cc}
\check{B}_{q}(x, \lambda) & A_{q}(x, \lambda) \\
-\check{C}_{q}(x, \lambda) & \check{A}_{q}(x, \lambda)
\end{array}\right) \text { if } \quad \Re(\lambda)<0 .
\end{aligned}
$$

Observe that for all $\lambda=i u \in i \mathbf{R}$, the following limits exist:

$$
\begin{aligned}
& M_{q}^{+}(x, i u):=\lim _{\lambda \rightarrow i u, \Re(\lambda)>0} M_{q}(x, \lambda)=\left(\begin{array}{cc}
A_{q}(x, i u) & -C_{q}(x, i u) \\
\check{A}_{q}(x, i u) & B_{q}(x, i u)
\end{array}\right), \\
& M_{q}^{-}(x, i u):=\lim _{\lambda \rightarrow i u, \Re(\lambda)<0} M_{q}(x, \lambda)=\left(\begin{array}{cc}
\check{B}_{q}(x, i u) & A_{q}(x, i u) \\
-\check{C}_{q}(x, i u) & \check{A}_{q}(x, i u)
\end{array}\right) .
\end{aligned}
$$

Theorem 4.2. For all $x \in] 0,+\infty\left[, q \in\left[0,+\infty\left[, \lambda \mapsto M_{q}(x, \lambda)\right.\right.\right.$ is the unique function satisfying the following properties.

1) $\lambda \mapsto M_{q}(x, \lambda)$ is analytic on the two half-planes $\{\Re(\lambda)>0\}$ and $\{\Re(\lambda)<0\}$.

2) $\lambda \mapsto M_{q}(x, \lambda)$ has a right limit $\left(M_{q}^{+}(x, i u)\right)$ and a left limit $\left(M_{q}^{-}(x, i u)\right)$ at every point $i u \in i \mathbf{R}$ and these two limits satisfy the equation

$$
M_{q}^{+}(x, i u)=M_{q}^{-}(x, i u)\left(\begin{array}{ll}
0 & -1 \\
1 & \phi(i u)+q
\end{array}\right) .
$$

3) The matrix

$$
\left(\begin{array}{cc}
\frac{M_{11}}{|\lambda|+1} & e^{\lambda x} M_{12} \\
e^{-\lambda x} M_{21} & \frac{M_{22}}{|\lambda|+1}
\end{array}\right)
$$

is bounded in $\{\Re(\lambda)>0\} \cup\{\Re(\lambda)<0\}$.

4) When $\Re(\lambda) \rightarrow-\infty$,

$$
\begin{aligned}
& \frac{M_{11}(x, \lambda)}{\check{\psi}_{0}(\lambda)} \rightarrow 1, \quad \check{\psi}_{0}(\lambda) M_{22}(x, \lambda) \rightarrow 1, \\
& \left.e^{\lambda x} M_{12}(x, \lambda) \rightarrow 0 \text { if }\right]-\infty, 0[\text { is regular },
\end{aligned}
$$

and

$$
\left.e^{-\lambda x} M_{21}(x, \lambda) \rightarrow 0 \text { if }\right]-\infty, 0[\text { is irregular, }
$$

and when $\Re(\lambda) \rightarrow+\infty$,

$$
\begin{aligned}
& \psi_{0}(\lambda) M_{11}(x, \lambda) \rightarrow 1, \quad \frac{M_{22}(x, \lambda)}{\psi_{0}(\lambda)} \rightarrow 1, \\
& \left.e^{-\lambda x} M_{21}(x, \lambda) \rightarrow 0 \text { if }\right] 0,+\infty[\text { is regular },
\end{aligned}
$$

and

$$
\left.e^{\lambda x} M_{12}(x, \lambda) \rightarrow 0 \text { if }\right] 0,+\infty[\text { is irregular. }
$$

\section{Connections With sCATtering theory}

The integral equations mentioned in Theorem 4.1 can be rewritten as a distribution-theoretic differential equation as follows:

$$
M_{q}^{\prime}(x, \lambda)=\left(\begin{array}{cl}
0 & e^{-\lambda x} \frac{H_{q}(d x)}{\check{H}_{q}(x)} \\
e^{\lambda x} \frac{\check{H}_{q}(d x)}{H_{q}\left(x^{-}\right)} & 0
\end{array}\right) M_{q}(x, \lambda),
$$


where $\lambda \in \mathbf{C} \backslash i \mathbf{R}$. This differential equation is a nonstandard form of the classical equation of the scattering theory on the line, with a measure-valued potential matrix

$$
\left(\begin{array}{cc}
0 & \frac{H_{q}(d x)}{\check{H}_{q}(x)} \\
\check{H}_{q}(d x) & 0
\end{array}\right) .
$$

Notice that these measures need not be finite.

We now review the basics of scattering theory, as expounded in [5. Suppose we are given a potential matrix $\left(\begin{array}{cc}0 & v(x) \\ \check{v}(x) & 0\end{array}\right)$, where $v$ and $\check{v}$ are real functions of a real variable satisfying some regularity assumptions; in particular, they are integrable. To this matrix is associated the differential equation

$$
Y^{\prime}(x, \lambda)=\left(\begin{array}{cl}
0 & e^{-\lambda x} v(x) \\
e^{\lambda x} \check{v}(x) & 0
\end{array}\right) Y(x, \lambda),
$$

where $\lambda$ is a complex parameter.

Denote by $J$ the matrix $J:=\left(\begin{array}{ll}1 & 0 \\ 0 & -1\end{array}\right)$. One can prove that for any imaginary complex number $\lambda=i u$ and any matrix solution $x \mapsto Y(x, i u)$, the matrix $e^{\frac{i u x}{2} J} Y(x, i u) e^{\frac{-i u x}{2} J}$ converges to the limits $y^{+}(i u)$ and $y^{-}(i u)$ as $x \rightarrow+\infty$ and $x \rightarrow-\infty$. The scattering matrix associated to the potential is defined by $S(i u)=\left[y^{-}(i u)\right]^{-1} y^{+}(i u)$. Obviously this matrix doesn't depend on the particular solution $x \mapsto Y(x, i u)$. On the other hand, for any complex $\lambda$, there exists a unique solution $x \mapsto \mathcal{Y}(x, \lambda)$ such that $e^{\frac{\lambda x}{2} J} \mathcal{Y}(x, \lambda) e^{\frac{-\lambda x}{2} J}$ converges to the identity matrix as $x \rightarrow-\infty$.

Shabat 21], see also [5, has proved that for any $x \in \mathbf{R}$, the function $\lambda \mapsto$ $\mathcal{Y}(x, \lambda)$ is analytic on the half-planes $\{\Re(\lambda)>0\}$ and $\{\Re(\lambda)<0\}$, has right limits $\left(\mathcal{Y}\left(x,(i u)^{+}\right)\right.$and left limits $\left(\mathcal{Y}\left(x,(i u)^{-}\right)\right.$at any point $i u$ on the imaginary axis and the jumps matrix $\left[\mathcal{Y}\left(x,(i u)^{-}\right]^{-1} \mathcal{Y}\left(x,(i u)^{+}\right)\right.$is equal to $S(i u)$. Moreover, $e^{\frac{\lambda x}{2} J} \mathcal{Y}(x, \lambda) e^{\frac{-\lambda x}{2} J}$ converges to the identity matrix as $|\lambda| \rightarrow+\infty$. It is then clear that the function $\lambda \mapsto \mathcal{Y}(x, \lambda)$ is entirely determined by these conditions. The determination of the matrix $\mathcal{Y}(x, \lambda)$ and consequently of the potential matrix from the scattering matrix ("the inverse problem") reduces to solving this Riemann-Hilbert problem.

In our problem, the coordinates of the matrix-valued function obtained by extending the potential matrix

$$
\left(\begin{array}{cc}
0 & \frac{H_{q}(d x)}{\check{H}_{q}(x)} \\
\frac{\check{H}_{q}(d x)}{H_{q}\left(x^{-}\right)} & 0
\end{array}\right)
$$

to zero on $]-\infty, 0[$ are measures instead of functions. Moreover, these measures are not finite unless $\mathbf{P}$ is the distribution of a compound Poisson process and $q$ is positive. Consequently, none of the solutions of the associated differential equation is regular both as $x \rightarrow+\infty$ and $x \rightarrow-\infty$; therefore, the matrices $y^{+}(i u), y^{-}(i u)$ and $S(i u)$ do not exist in the sense given above. Moreover, the differential equation $x \mapsto Y(x, \lambda)$ need not have a solution such that the matrix $e^{\frac{\lambda x}{2} J} Y(x, \lambda) e^{\frac{-\lambda x}{2} J}$ converges to the identity matrix as $|\lambda|$ goes to $+\infty$. The solution of the differential equation $(S c)$ we chose is the most convenient from a probabilistic point of view. Theorem 4.2 tells us that this matrix is the solution of a Riemann-Hilbert problem, 
as in classical scattering theory. In the present setting the role of the scattering matrix is played by the matrix $\left(\begin{array}{ll}0 & -1 \\ 1 & \phi(i u)+q\end{array}\right)$.

\section{Connection with the exit PRoblem}

We now explain how the resolvent of the Markov process $(X, S, I)$ and the distributions of the position of this process at different times are related to the six functions $A, \breve{A}, C, \breve{C}, B, \breve{B}$. Denote by $\operatorname{supp}(L)$ and $\operatorname{supp}(\check{L})$ the supports of the random measures $L(d t)$ and $\check{L}(d t)$, and, for all positive times $t$,

$$
g_{t}:=\sup \left(\left[0, t[\cap \operatorname{supp}(L)), \quad \check{g}_{t}:=\sup ([0, t[\cap \operatorname{supp}(\check{L})) .\right.\right.
$$

Proposition 6.1. For all $\lambda_{1}, \lambda_{2} \in \mathbf{C}, q_{1}, q_{2} \in[0,+\infty[, x \in] 0,+\infty[$, one has

$$
\begin{aligned}
& \check{A}_{q_{1}}\left(x, \lambda_{1}\right) A_{q_{2}}\left(x, \lambda_{2}\right)=\mathbf{P}\left(\int_{[0,+\infty[} e^{-\lambda_{1} I_{t}-q_{1} \check{g}_{t}} 1_{S_{t}-I_{t} \leq x} e^{-\lambda_{2}\left(X_{t}-I_{t}\right)-q_{2}\left(t-\check{g}_{t}\right)} d t\right) \\
& \quad=\mathbf{P}\left(\int_{[0,+\infty[} e^{-\lambda_{2} S_{t}-q_{2} g_{t}} 1_{S_{t}-I_{t} \leq x} e^{-\lambda_{1}\left(X_{t}-S_{t}\right)-q_{1}\left(t-g_{t}\right)} d t\right) .
\end{aligned}
$$

For $q_{1}=q_{2}=q$, the previous statement shows that the pair of functions $\left(A_{q}, \check{A}_{q}\right)$ determines the value of $U_{q}(0,0,0, d y d u d v)$, where $U$ is the resolvent of the trivariate Markov process $(X, S, I)$. (One can easily deduce from this the value of $U_{q}(x, a, b, d y d u d v)$ for any triple $(x, a, b)$.) We shall see that this result is an immediate consequence of a path decomposition stated in Proposition 7.1 and we will prove it later. Notice the limits

$$
\lim _{x \rightarrow+\infty} \check{A}_{q_{1}}\left(x, \lambda_{1}\right)=\frac{1}{\check{\psi}_{q_{1}}\left(\lambda_{1}\right)} \quad \Re\left(\lambda_{1}\right) \leq 0, q_{1} \in\left[0,+\infty\left[, q_{1} \Re\left(\lambda_{1}\right) \neq 0\right.\right.
$$

and

$$
\lim _{x \rightarrow+\infty} A_{q_{2}}\left(x, \lambda_{2}\right)=\frac{1}{\psi_{q_{2}}\left(\lambda_{2}\right)} \quad \Re\left(\lambda_{2}\right) \geq 0, q_{2} \in\left[0,+\infty\left[, q_{2} \Re\left(\lambda_{2}\right) \neq 0 .\right.\right.
$$

Thus, the limit of the identity of Proposition 6.1, as $x$ goes to $+\infty$, gives us a less precise version of theorem $1 \mathrm{~F}$ in Bingham's paper [4] and for $\lambda_{1}$ and $\lambda_{2}$ on the imaginary axis, it is also a less precise version of theorem 49.1 in Sato's book [20]. These two versions of Wiener-Hopf factorization seem to be the most precise which have been obtained until now.

The next propositions are immediate consequences of the definition of our functions $A, B, C, \check{A}, \check{B}, \check{C}$ and of the compensation formula stated in Proposition 3.1 The proofs are left to the reader.

Proposition 6.2. For all $\Re\left(\mu_{1}\right) \geq 0, \Re\left(\mu_{2}\right) \leq 0, q_{1}, q_{2} \in[0,+\infty[, x \in] 0,+\infty[$,

$$
\begin{array}{r}
\mathbf{P}\left(\exp \left(-\mu_{1} S_{V_{x}}-q_{1} g_{V_{x}}-\mu_{2}\left(X_{V_{x}}-S_{V_{x}}\right)-q_{2}\left(V_{x}-g_{V_{x}}\right)\right)\right) \\
=\frac{1}{B_{q_{1}}\left(x, \mu_{1}\right)} \check{C}_{q_{2}}\left(x, \mu_{2}\right), \\
\mathbf{P}\left(\exp \left(-\mu_{2} I_{V^{x}}-q_{1} \check{g}_{V^{x}}-\mu_{1}\left(X_{V^{x}}-I_{V^{x}}\right)-q_{2}\left(V^{x}-\check{g}_{V^{x}}\right)\right)\right) \\
=\frac{1}{\check{B}_{q_{1}}\left(x, \mu_{2}\right)} C_{q_{2}}\left(x, \mu_{1}\right) .
\end{array}
$$


When $X$ is a stable Lévy process and $q=0$, one can use the identities above and the explicit expressions of the functions $B_{0}, \check{B}_{0}, C_{0}, \check{C}_{0}$ given in Section 12 of this paper in order to recover Kyprianou's results [13. When $X$ has no positive jumps, using the same machinery, one can derive the results of Avram, Kyprianou, and Pistorius, 1], Kyprianou and Palmowski [14, Doney [6], Nguyen-Ngoc and Yor [15, and Pistorius [16] and [17].

Let us denote by

$$
U^{x}:=\inf \left\{t \mid S_{t}-I_{t} \geq x \text { and } S_{t}=X_{t}\right\} \wedge \inf \left\{t \mid S_{t}-I_{t}>x \text { and } X_{t}=I_{t}\right\}
$$

Proposition 6.3. For all $\lambda \in \mathbf{C}, \Re\left(\mu_{1}\right) \geq 0, \Re\left(\mu_{2}\right) \leq 0, q_{1}, q_{2} \in[0,+\infty[, x \in$ ] $0,+\infty[$,

$$
\begin{array}{r}
\mathbf{P}\left(\exp \left(-\lambda I_{U^{x}}-q_{1} \check{g}_{U^{x}}-\mu_{1}\left(X_{U^{x}}-I_{U^{x}}\right)-q_{2}\left(U^{x}-\check{g}_{U^{x}}\right)\right) ; S_{U^{x}}=X_{U^{x}}\right) \\
=\check{A}_{q_{1}}(x, \lambda) C_{q_{2}}\left(x^{-}, \mu_{1}\right), \\
\mathbf{P}\left(\exp \left(-\lambda S_{U_{x}}-q_{1} g_{U^{x}}-\mu_{2}\left(X_{U^{x}}-S_{U^{x}}\right)-q_{2}\left(U^{x}-g_{U^{x}}\right)\right) ; I_{U^{x}}=X_{U^{x}}\right) \\
=A_{q_{1}}\left(x^{-}, \lambda\right) \check{C}_{q_{2}}\left(x, \mu_{2}\right) .
\end{array}
$$

Despite this nice path decomposition, the behavior of the process $(X, S, I)$ at time $U^{x}$ doesn't seem to have been investigated thus far.

Let $A_{q}(x, d y)$ be the measure with Laplace transform $A_{q}(x, \lambda)\left(A_{q}(x, d y):=\right.$ $\left.\mathbf{P}\left(\int_{0}^{+\infty} 1_{S_{t}-I_{t} \leq x} 1_{S_{t} \in d y} e^{-q t} L(d t)\right)\right)$. This measure is absolutely continuous with respect to the measure $A_{q}(+\infty, d y):=\mathbf{P}\left(\int_{[0,+\infty[} 1_{S_{t} \in d y} e^{-q t} L(d t)\right)$, with a density $\alpha_{q}(x, y)$. This entitles us to define

$$
A_{q}(b+y, d y):=\alpha_{q}(b+y, y) A_{q}(+\infty, d y)=\mathbf{P}\left(\int_{[0,+\infty[} 1_{-I_{t} \leq b} 1_{S_{t} \in d y} e^{-q t} L(d t)\right) .
$$

Similarly, denote by $\check{A}_{q}(a-y, d y)$ the measure

$$
\check{A}_{q}(a-y, d y):=\mathbf{P}\left(\int_{[0,+\infty[} 1_{S_{t} \leq a} 1_{I_{t} \in d y} e^{-q t} \check{L}(d t)\right) .
$$

For all positive reals $a, b$, denote by $T_{b}^{a}:=\inf \left\{t \mid X_{t} \notin[-b, a]\right\}$.

Proposition 6.4. For all $\lambda \in \mathbf{C}, \Re\left(\mu_{1}\right) \geq 0, \Re\left(\mu_{2}\right) \leq 0, q_{1}, q_{2} \in[0,+\infty[$,

$$
\begin{array}{r}
\mathbf{P}\left(\exp \left(-\lambda I_{T_{b}^{a}}-q_{1} \check{g}_{T_{b}^{a}}-\mu_{1}\left(X_{T_{b}^{a}}-I_{T_{b}^{a}}\right)-q_{2}\left(T_{b}^{a}-\check{g}_{T_{b}^{a}}\right)\right) ; X_{T_{b}^{a}}=S_{T_{b}^{a}}\right) \\
=\int_{[-b, 0]} e^{-\lambda y} C_{q_{2}}\left(a-y, \mu_{1}\right) \check{A}_{q_{1}}(a-y, d y), \\
\mathbf{P}\left(\exp \left(-\lambda S_{T_{b}^{a}}-q_{1} g_{T_{b}^{a}}-\mu_{2}\left(X_{T_{b}^{a}-} S_{T_{b}^{a}}\right)-q_{2}\left(T_{b}^{a}-g_{T_{b}^{a}}\right)\right) ; X_{T_{b}^{a}}=I_{T_{b}^{a}}\right) \\
=\int_{[0, a]} e^{-\lambda y} \check{C}_{q_{2}}\left(b+y, \mu_{2}\right) A_{q_{1}}(b+y, d y) .
\end{array}
$$


Again, when $X$ is a stable Lévy process and $q=0$, one can use the identities below and the explicit expressions of the functions $A_{0}, \check{A}_{0}, C_{0}, \check{C}_{0}$ presented in Section 12 of this paper and recover Rogozin's 19 results. When $X$ has no positive jumps, using the same machinery, one can obtain the results of Takács 24] (see also Emery [7] and Rogers [18 for a simpler proof) and more detailed results in Koryluk, Suprun and Shurenkov [12, Suprun [23] and Bertoin [2].

\section{INDEPENDENCE OF PAST AND POST MINIMUM PROCESSES "CONDITIONALlY ON THE AMPLITUdE" AND PROOF OF PROPOSITION 6.1}

The results of this section can be found in the author's preprint 9 .

We first introduce some more notation. For all $q \in\left[0,+\infty\left[\right.\right.$, let $\mathbf{Q}_{q}, \mathbf{Q}_{q}^{\uparrow}$ and $\mathbf{Q}_{q}^{\downarrow}$ be the measures on $(\Omega, \mathcal{F}, \mathbf{P})$ defined as follows:

$$
\begin{aligned}
& \mathbf{Q}_{q}(d w):=\mathbf{P}\left[\int_{0}^{+\infty} 1_{\left(X_{s}\right)_{0 \leq s<t} \in d w} e^{-q t} d t\right], \\
& \mathbf{Q}_{q}^{\uparrow}(d w):=\mathbf{P}\left[\int_{[0,+\infty[} 1_{\left(S_{t}-X_{(t-s)^{-}} ; s \geq 0\right) \in d w} e^{-q t} L(d t)\right], \\
& \mathbf{Q}_{q}^{\downarrow}(d w):=\mathbf{P}\left[\int_{[0,+\infty[} 1_{\left(I_{t}-X_{(t-s)^{-}} ; s \geq 0\right) \in d w} e^{-q t} \check{L}(d t)\right] .
\end{aligned}
$$

All these measures are supported by the set of paths with finite lifetime. Notice that for a positive $q, \mathbf{Q}_{q}, \mathbf{Q}_{q}^{\uparrow}$ and $\mathbf{Q}_{q}^{\downarrow}$ are finite measures and $\mathbf{P}_{q}:=q \mathbf{Q}_{q}$ is the distribution of the Lévy process $X$ under $\mathbf{P}$ killed at an independent exponential time with parameter $q$. Notice also that the measure $\mathbf{Q}_{0}^{\uparrow}$ is finite if $\lim _{t \rightarrow+\infty} X_{t}=$ $-\infty$ (P-a.s.), and $\mathbf{Q}_{0}^{\downarrow}$ is finite if $\lim _{t \rightarrow+\infty} X_{t}=+\infty$ ( $\mathbf{P}$-a.s.). The measures $\mathbf{Q}_{0}^{\uparrow}$ and $\mathbf{Q}_{0}^{\downarrow}$ are infinite in the other cases and $\mathbf{Q}_{0}$ is infinite in all cases.

Let $\zeta$ be the lifetime of the canonical process $X$, and let $F, M, m$ be the final values of $X, S, I$,

$$
\zeta:=\sup \left\{t, X_{t} \in \mathbf{R}\right\} \quad F:=X_{\zeta-} \quad M:=S_{\zeta-} \quad m:=I_{\zeta-} .
$$

Denote by $\sigma$ and $\rho$ respectively the last time when $X$ takes its maximal value and the first time when $X$ takes its minimal value $\left(X_{0-}:=0\right)$ :

$$
\sigma:=\sup \left\{t<\zeta \mid X_{t} \vee X_{t-}=M\right\}, \quad \rho:=\inf \left\{t \geq 0 \mid X_{t} \wedge X_{t-}=m\right\} .
$$

Also $\zeta, \sigma, \rho, F, M, m$ are assumed to be zero when the path is constantly equal to the cemetery point $\delta$.

Notice that, under $\mathbf{Q}_{q}$, for every $q \in[0,+\infty[$, the time $\sigma$ (respectively $\rho$ ) is respectively the unique time at which the process $X_{t} \vee X_{t-}\left(\right.$ resp. $\left.X_{t} \wedge X_{t-}\right)$ takes its maximal (resp. minimal) value, unless $\mathbf{P}$ is the distribution of a compound Poisson process.

Notice that $\frac{\mathbf{Q}_{q}^{\uparrow}(d w)}{\mathbf{Q}_{q}^{\uparrow}(\Omega)}$ and $\frac{\mathbf{Q}_{q}^{\downarrow}(d w)}{\mathbf{Q}_{q}^{\downarrow}(\Omega)}$ are respectively the law of the process ( $M-$ $\left.X_{(\sigma-s)^{-}}, s \geq 0\right)$ and the law of $\left(m-X_{(\rho-s)^{-}} ; s \geq 0\right)$ under $\mathbf{P}_{q}=q \mathbf{Q}_{q}$. This property is a standard consequence of the compensation formula of Proposition 3.1. 
The next identities follow straightforwardly from the definitions:

$$
\begin{aligned}
& A_{q}(x, \lambda)=\mathbf{Q}_{q}^{\uparrow}\left(e^{-\lambda F} ; M \leq x\right)=\mathbf{Q}_{0}^{\uparrow}\left(e^{-\lambda F-q \zeta} ; M \leq x\right), \\
& H_{q}(x)=\mathbf{Q}_{q}^{\uparrow}(M \leq x)=\mathbf{Q}_{0}^{\uparrow}\left(e^{-q \zeta} ; M \leq x\right), \\
& \frac{1}{\psi_{q}(\lambda)}=\mathbf{Q}_{q}^{\uparrow}\left(e^{-\lambda F}\right)=\mathbf{Q}_{0}^{\uparrow}\left(e^{-\lambda F-q \zeta}\right), \\
& \check{A}_{q}(x, \lambda)=\mathbf{Q}_{q}^{\downarrow}\left(e^{-\lambda F} ;-m \leq x\right)=\mathbf{Q}_{0}^{\downarrow}\left(e^{-\lambda F-q \zeta} ;-m \leq x\right), \\
& \frac{1}{\check{\psi}_{q}(\lambda)}=\mathbf{Q}_{q}^{\downarrow}\left(e^{-\lambda F}\right)=\mathbf{Q}_{0}^{\downarrow}\left(e^{-\lambda F-q \zeta}\right), \\
& \check{H}_{q}(x)=\mathbf{Q}_{q}^{\downarrow}(-m \leq x)=\mathbf{Q}_{0}^{\downarrow}\left(e^{-q \zeta} ;-m \leq x\right) .
\end{aligned}
$$

Proposition 7.1. For every $q \in[0,+\infty[, x \in] 0,+\infty]$,

$$
\begin{aligned}
& \mathbf{Q}_{q}\left(\left(m-X_{(\rho-t)^{-}} ; t \geq 0\right) \in d w_{1} ; M-m \leq x,\left(X_{t+\rho}-m ; t \geq 0\right) \in d w_{2}\right) \\
& \quad=\mathbf{Q}_{q}\left(\left(M-X_{(\sigma-t)^{-}} ; t \geq 0\right) \in d w_{2} ; M-m \leq x,\left(X_{t+\sigma}-M ; t \geq 0\right) \in d w_{1}\right) \\
& \quad=\mathbf{Q}_{q}^{\downarrow}\left(d w_{1} ;-m \leq x\right) \mathbf{Q}_{q}^{\uparrow}\left(d w_{2} ; M \leq x\right) .
\end{aligned}
$$

Proof. Let us first prove the assertions for $q>0$ and $x=+\infty$. One has the following identities:

$$
\begin{aligned}
& \mathbf{P}_{q}\left(\left(m-X_{(\rho-t)^{-}} ; t \geq 0\right) \in d w_{1} ;\left(X_{t+\rho}-m ; t \geq 0\right) \in d w_{2}\right) \\
& \quad=\mathbf{P}_{q}\left(\left(m-X_{(\rho-t)^{-}} ; t \geq 0\right) \in d w_{1}\right) \mathbf{P}_{q}\left(\left(X_{t+\rho}-m ; t \geq 0\right) \in d w_{2}\right) \\
& \quad=\mathbf{P}_{q}\left(\left(m-X_{(\rho-t)^{-}} ; t \geq 0\right) \in d w_{1}\right) \mathbf{P}_{q}\left(\left(M-X_{(\sigma-t)^{-}}, t \geq 0\right) \in d w_{2}\right) \\
& \quad=\frac{\mathbf{Q}_{q}^{\downarrow}\left(d w_{1}\right)}{\mathbf{Q}_{q}^{\downarrow}(\Omega)} \frac{\mathbf{Q}_{q}^{\uparrow}\left(d w_{2}\right)}{\mathbf{Q}_{q}^{\uparrow}(\Omega)} .
\end{aligned}
$$

The first equality follows from the well-known independence of the past and post minimum processes (see 3, lemma 6, chapter 6, for example), the second from the fact, again well known (see [3, lemma 2, chapter 2) that the process $\left(F-X_{(\zeta-t)^{-}} ; t \geq 0\right)$ has the same law as $X$ under $\mathbf{P}_{q}$. The last one follows from the compensation formula, as we have already mentioned.

On the other hand, we obtain

$$
\mathbf{Q}_{q}^{\downarrow}(\Omega) \mathbf{Q}_{q}^{\uparrow}(\Omega)=\frac{1}{\check{\psi}_{q}(0)} \frac{1}{\psi_{q}(0)}=\frac{1}{q} .
$$

The first identity follows from the definitions of $\mathbf{Q}_{q}^{\downarrow}$ and $\mathbf{Q}_{q}^{\uparrow}$, the second one from the Wiener-Hopf equation of Proposition 2.1 for $i u=0$.

Recall that $\mathbf{P}_{q}=q \mathbf{Q}_{q}$ and multiply the identity (7.1) by $\frac{1}{q}$ and use (7.2) to get

$$
\mathbf{Q}_{q}\left(\left(m-X_{(\rho-t)^{-}} ; t \geq 0\right) \in d w_{1} ;\left(X_{t+\rho}-m ; t \geq 0\right) \in d w_{2}\right)=\mathbf{Q}_{q}^{\downarrow}\left(d w_{1}\right) \mathbf{Q}_{q}^{\uparrow}\left(d w_{2}\right) \text {. }
$$

Letting $q$ go to 0 , we get the same identity for $q=0$.

The event $\{M-m \leq x\}$ can be written as the following intersection of two independent events:

$$
\{M-m \leq x\}=\left\{-\min \left[m-X_{(\rho-t)^{-}} ; t \geq 0\right] \leq x\right\} \cap\left\{\max \left[X_{t+\rho}-m ; t \geq 0\right] \leq x\right\} .
$$

This yields

$$
\begin{aligned}
& \mathbf{Q}_{q}\left(\left(m-X_{(\rho-t)^{-}} ; t \geq 0\right) \in d w_{1} ; M-m \leq x ;\left(X_{t+\rho}-m ; t \geq 0\right) \in d w_{2}\right) \\
& \quad=\mathbf{Q}_{q}^{\downarrow}\left(d w_{1} ;-m \leq x\right) \mathbf{Q}_{q}^{\uparrow}\left(d w_{2} ; M \leq x\right) .
\end{aligned}
$$


Using the identity in law of the process $\left(F-X_{(\zeta-t)^{-}} ; t \geq 0\right)$ and $X$, we obtain the other identity of Proposition 7.1 .

Proof of Proposition 6.1. Let $X^{\uparrow}$ and $X^{(-)}$be the processes $\left(X_{t+\rho}-m ; t \geq 0\right)$ and $\left(m-X_{(\rho-t)^{-}} ; t \geq 0\right)$, respectively. Then,

$$
\begin{aligned}
\mathbf{P} & \left(\int_{[0,+\infty[} e^{-\lambda_{1} I_{t}-q_{1} \check{g}_{t}} 1_{S_{t}-I_{t} \leq x} e^{-\lambda_{2}\left(X_{t}-I_{t}\right)-q_{2}\left(t-\check{g}_{t}\right)} d t\right) \\
& =\mathbf{Q}_{0}\left(e^{-\lambda_{1} m-q_{1} \rho} 1_{M-m \leq x} e^{-\lambda_{2}(F-m)-q_{2}(\zeta-\rho)}\right) \\
& =\mathbf{Q}_{0}\left(e^{-\lambda_{1} F\left(X^{(-)}\right)-q_{1} \zeta\left(X^{(-)}\right)} 1_{-m\left(X^{(-)}\right) \leq x} 1_{M\left(X^{\uparrow}\right) \leq x} e^{-\lambda_{2} F\left(X^{\uparrow}\right)-q_{2} \zeta\left(X^{\uparrow}\right)}\right) \\
& =\mathbf{Q}_{0}^{\downarrow}\left(e^{-\lambda_{1} F-q_{1} \zeta} ;-m \leq x\right) \mathbf{Q}_{0}^{\uparrow}\left(e^{-\lambda_{2} F-q_{2} \zeta} ; M \leq x\right)=\check{A}_{q_{1}}\left(x, \lambda_{1}\right) A_{q_{2}}\left(x, \lambda_{2}\right) .
\end{aligned}
$$

The first, the second and the fourth identities follow from the definitions. The third one follows from Proposition 7.1

Similarly, the second assertion of Proposition 6.1 follows from the second assertion of Proposition 7.1 ,

Unless explicitly specified, all the statements made in the rest of the paper hold for every $q \in[0,+\infty[$.

\section{Proof of property 1) of Theorem 4.1}

Write for all $x \in] 0,+\infty[$,

$$
\mathbf{Q}_{q}^{\downarrow x}(d w):=\mathbf{Q}_{q}^{\downarrow}(d w \mid-m \leq x), \quad \mathbf{Q}_{q}^{\uparrow x}(d w):=\mathbf{Q}_{q}^{\uparrow}(d w \mid M<x) .
$$

Notice that when $\mathbf{Q}_{q}^{\downarrow}(m=0)>0$, the measure $\mathbf{Q}_{q}^{\downarrow}(d w \mid-m=0)$ is the Dirac mass on the path constantly equal to $\delta$. We make the convention that $\mathbf{Q}_{q}^{\downarrow 0}$ and $\mathbf{Q}_{q}^{\uparrow 0}$ are this Dirac mass.

\section{Lemma 8.1.}

$$
\begin{aligned}
& \mathbf{Q}_{q}^{\uparrow}\left(\left(X_{\sigma+t}-M ; t \geq 0\right) \in d w \mid M-X_{(\sigma-t)^{-}} ; t \geq 0\right)=\mathbf{Q}_{q}^{\downarrow M}(d w), \\
& \mathbf{Q}_{q}^{\downarrow}\left(\left(X_{\rho+t}-m ; t \geq 0\right) \in d w \mid m-X_{(\rho-t)^{-}} ; t \geq 0\right)=\mathbf{Q}_{q}^{\uparrow-m}(d w) .
\end{aligned}
$$

Proof. Still denote by $X^{\uparrow}$ and $X^{(-)}$the processes $\left(X_{t+\rho}-m ; t \geq 0\right)$ and $\left(m-X_{(\rho-t)^{-}} ; t \geq 0\right)$, respectively. Using the identities of Proposition 7.1 with $x=+\infty$, we deduce that these two processes are independent under $\mathbf{Q}_{q}$. We then obtain (remember that $\check{H}_{q}\left(x^{-}\right)=\mathbf{Q}_{q}^{\downarrow}(-m<x)$ )

$$
\begin{gathered}
\check{H}_{q}\left(x^{-}\right) \cdot \mathbf{Q}_{q}^{\uparrow}\left(\left(M-X_{(\sigma-t)^{-}} ; t \geq 0\right) \in d w_{0}, M \in d x,\left(X_{\sigma+t}-M ; t \geq 0\right) \in d w\right) \\
=\mathbf{Q}_{q}\left(-m\left[X^{(-)}\right]<x ;\left(M-X_{(\sigma-t)^{-}} ; t \geq 0\right)\left[X^{\uparrow}\right] \in d w_{0}\right. \\
\left.M\left[X^{\uparrow}\right] \in d x,\left(X_{\sigma+t}-M ; t \geq 0\right)\left[X^{\uparrow}\right] \in d w\right) .
\end{gathered}
$$

Notice the identities between random variables $-m\left[X^{(-)}\right]=M\left[X_{t} ; t \in[0, \rho[]-m\right.$ and $M\left[X^{\uparrow}\right]=M\left[X_{t} ; t \geq \rho\right]-m$. Thus, on the event $\left\{-m\left[X^{(-)}\right]<x ; M\left[X^{\uparrow}\right] \in d x\right\}$, we have $\rho \leq \sigma$ and $m=I_{\sigma^{-}}$; moreover, the time $\rho_{\sigma}:=\inf \left\{t ; X_{t} \wedge X_{t^{-}}=I_{\sigma^{-}}\right\}$is 
equal to $\rho$. We then obtain the identities of events

$$
\begin{aligned}
&\left\{-m\left[X^{(-)}\right]<x ;\left(M-X_{(\sigma-t)^{-}} ; t \geq 0\right)\left[X^{\uparrow}\right] \in d w_{0}, M\left[X^{\uparrow}\right] \in d x,\right.\left.\left(X_{\sigma+t}-M ; t \geq 0\right)\left[X^{\uparrow}\right] \in d w\right\} \\
&=\left\{S_{\rho^{-}}-m<x,\left(M-X_{(\sigma-t)^{-}} ; t \in\left[0, \sigma-\rho[) \in d w_{0},\right.\right.\right. \\
&\left.M-m \in d x,\left(X_{\sigma+t}-M ; t \geq 0\right) \in d w\right\} \\
&=\left\{S_{\rho_{\bar{\sigma}}}-I_{\sigma^{-}}<x ;\left(M-X_{(\sigma-t)^{-}} ; t \in\left[0, \sigma-\rho_{\sigma}[) \in d w_{0},\right.\right.\right. \\
&\left.M-I_{\sigma^{-}} \in d x,-m\left[X_{\sigma+t}-M ; t \geq 0\right] \leq x,\left(X_{\sigma+t}-M ; t \geq 0\right) \in d w\right\} .
\end{aligned}
$$

On plugging these identities into (8.1), and using the independence property of the processes $\left(M-X_{(\sigma-t)^{-}} ; t \geq 0\right)$ and $\left(X_{\sigma+t}-M ; t \geq 0\right)$ under $\mathbf{Q}_{q}$ as stated in Proposition 7.1, we obtain

$$
\begin{gathered}
\check{H}_{q}\left(x^{-}\right) \cdot \mathbf{Q}_{q}^{\uparrow}\left(\left(M-X_{(\sigma-t)^{-}} ; t \geq 0\right) \in d w_{0}, M \in d x,\left(X_{\sigma+t}-M ; t \geq 0\right) \in d w\right) \\
=\mathbf{Q}_{q}\left(S_{\rho_{\bar{\sigma}}^{-}}-I_{\sigma^{-}}<x ;\left(M-X_{(\sigma-t)^{-}} ; t \in\left[0, \sigma-\rho_{\sigma}[) \in d w_{0}, M-I_{\sigma^{-}} \in d x,\right.\right.\right. \\
\left.-m\left[X_{\sigma+t}-M ; t \geq 0\right] \leq x\right) \mathbf{Q}_{q}^{\downarrow x}(d w) .
\end{gathered}
$$

Integrating this identity with respect to $d w$, we obtain

$$
\begin{aligned}
& \check{H}_{q}\left(x^{-}\right) \cdot \mathbf{Q}_{q}^{\uparrow}\left(\left(M-X_{(\sigma-t)^{-}} ; t \geq 0\right) \in d w_{0}, M \in d x\right) \\
& =\mathbf{Q}_{q}\left(S_{\rho_{\bar{\sigma}}}-I_{\sigma^{-}}<x,\left(M-X_{(\sigma-t)^{-}} ; t \in\left[0, \sigma-\rho_{\sigma}[) \in d w_{0}, M-I_{\sigma^{-}} \in d x\right.\right.\right. \\
& \left.-m\left[X_{\sigma+t}-M ; t \geq 0\right] \leq x\right)
\end{aligned}
$$

Identities (8.2) and (8.3) then give

$$
\begin{aligned}
& \check{H}_{q}\left(x^{-}\right) \cdot \mathbf{Q}_{q}^{\uparrow}\left(\left(M-X_{(\sigma-t)^{-}} ; t \geq 0\right) \in d w_{0}, M \in d x,\left(X_{\sigma+t}-M ; t \geq 0\right) \in d w\right) \\
& \quad=\check{H}_{q}\left(x^{-}\right) \cdot \mathbf{Q}_{q}^{\uparrow}\left(\left(M-X_{(\sigma-t)^{-}} ; t \geq 0\right) \in d w_{0}, M \in d x\right) \mathbf{Q}_{q}^{\downarrow x}(d w) .
\end{aligned}
$$

When $x$ is positive, $\check{H}_{q}\left(x^{-}\right)$is positive and we can divide by $\check{H}_{q}\left(x^{-}\right)$the previous identity. We then obtain the first assertion of the lemma. When $x=0$, this assertion is trivial.

We proceed similarly to obtain the second assertion of the lemma.

Proof of fact 1) of Theorem 4.1 For all $x \in] 0,+\infty[$ and $\lambda \in \mathbf{C}$,

$$
\begin{aligned}
A_{q}\left(x^{-}, \lambda\right) & =\mathbf{Q}_{q}^{\uparrow}\left(M<x, e^{-\lambda F}\right)=H_{q}(0)+\int_{] 0, x[} \mathbf{Q}_{q}^{\uparrow}\left(e^{-\lambda F} \mid M=y\right) H_{q}(d y) \\
& =H_{q}(0)+\int_{] 0, x[} e^{-\lambda y} \mathbf{Q}_{q}^{\uparrow}\left(e^{-\lambda(F-M)} \mid M=y\right) H_{q}(d y) \\
& =H_{q}(0)+\int_{] 0, x[} e^{-\lambda y} \mathbf{Q}_{q}^{\uparrow}\left(e^{-\lambda F\left[X_{\sigma+t}-M ; t \geq 0\right]} \mid M=y\right) H_{q}(d y) \\
& =H_{q}(0)+\int_{] 0, x[} e^{-\lambda y} \mathbf{Q}_{q}^{\downarrow}\left(e^{-\lambda F} \mid-m \leq y\right) H_{q}(d y) \\
& =H_{q}(0)+\int_{] 0, x[} e^{-\lambda y} \mathbf{Q}_{q}^{\downarrow}\left(e^{-\lambda F} ;-m \leq y\right) \frac{H_{q}(d y)}{\check{H}_{q}(y)} \\
& =H_{q}(0)+\int_{] 0, x[} e^{-\lambda y} \check{A}_{q}(y, \lambda) \frac{H_{q}(d y)}{H_{q}(y)} .
\end{aligned}
$$

The fourth identity follows from Lemma 8.1 the others are obvious.

The identity

$$
\check{A}_{q}(x, \lambda)=\check{H}_{q}(0)+\int_{] 0, x]} e^{\lambda y} A_{q}\left(y^{-}, \lambda\right) \frac{\check{H}_{q}(d y)}{H_{q}\left(y^{-}\right)}
$$

is proved in a similar way. 


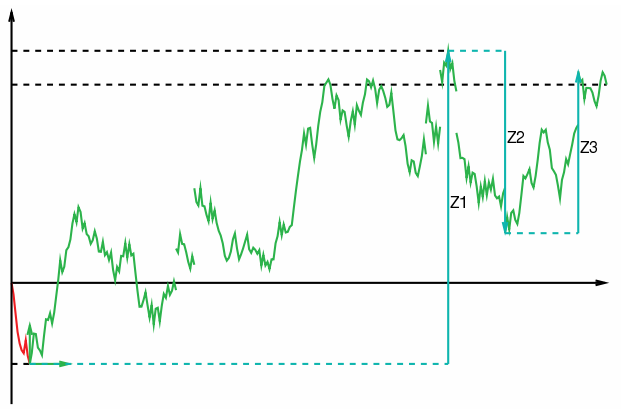

FiguRE 1

9. Proof of properties 2 And 3 of Theorem 4.1

9.1. Two Markov chains. We now define the successive minima and maxima. We first let

$$
\begin{array}{r}
M_{1}:=M \quad \text { and } \quad T_{1}:=\sigma \\
m_{2}:=\inf \left\{X_{t} \mid t \geq T_{1}\right\} \quad \text { and } \quad T_{2}:=\inf \left\{t \geq T_{1} \mid X_{t} \wedge X_{t-}=m_{2}\right\} .
\end{array}
$$

Then we define inductively

$$
\begin{aligned}
M_{2 n+1}:=\sup \left\{X_{t} \mid t \geq T_{2 n}\right\} \quad \text { and } \quad T_{2 n+1}:=\sup \left\{t \geq T_{2 n} \mid X_{t} \vee X_{t-}=M_{2 n+1}\right\}, \\
m_{2 n+2}:=\inf \left\{X_{t} \mid t \geq T_{2 n+1}\right\} \quad \text { and } \quad T_{2 n+2}:=\inf \left\{t \geq T_{2 n+1} \mid X_{t} \wedge X_{t-}=m_{2 n+2}\right\}, \\
Z_{2 n+1}:=M_{2 n+1}-m_{2 n} \quad \text { and } \quad Z_{2 n+2}:=m_{2 n+2}-M_{2 n+1} .
\end{aligned}
$$

If $T_{n}=\zeta$, put $Z_{n+1}:=0$ and $T_{n+1}:=\zeta$. Notice that $F=\sum_{1}^{+\infty} Z_{n}$.

The behavior of the sequence $\left(Z_{n}\right)$ is depicted in Figure 1. Similarly, let

$$
\begin{aligned}
m_{1}:=m & \text { and } S_{1}:=\rho \\
M_{2}:=\sup \left\{X_{t} \mid t \geq T_{1}\right\} & \text { and } \quad S_{2}:=\sup \left\{t \geq T_{1} \mid X_{t} \vee X_{t-}=I_{2}\right\} \\
m_{2 n+1}:=\inf \left\{X_{t} \mid t \geq S_{2 n}\right\} & \text { and } S_{2 n+1}:=\inf \left\{t \geq S_{2 n} \mid X_{t} \wedge X_{t-}=m_{2 n+1}\right\} \\
M_{2 n+2}:=\sup \left\{X_{t} \mid t \geq S_{2 n+1}\right\} & \text { and } \quad S_{2 n+2}:=\sup \left\{t \geq S_{2 n+1} \mid X_{t} \vee X_{t-}=M_{2 n+2}\right\}
\end{aligned}
$$

and

$$
Y_{2 n+1}:=m_{2 n+1}-M_{2 n} \quad \text { and } \quad Y_{2 n+2}:=M_{2 n+2}-m_{2 n+1} .
$$

If $S_{n}=\zeta$, put $Y_{n+1}:=0$ and $S_{n+1}:=\zeta$. Notice that $F=\sum_{1}^{+\infty} Y_{n}$.

Lemma 9.1. Under $\mathbf{Q}_{q}^{\uparrow}$ the sequence $\left(Z_{1}, Z_{2}, \ldots\right)$ is a Markov chain with transition kernel given by

$$
\begin{gathered}
\text { for } x \neq 0, \quad P_{q}(x, d y)=\frac{H_{q}(d y)}{H_{q}\left((-x)^{-}\right)} 1_{y \in[0,-x[}+\frac{\check{H}_{q}(-d y)}{\check{H}_{q}(x)} 1_{y \in[-x, 0]}, \\
P_{q}(0,\{0\})=1,
\end{gathered}
$$

and with initial law :

$$
\mathbf{Q}_{q}^{\uparrow}\left(Z_{1} \in d y\right)=H_{q}(d y)
$$

Under $\mathbf{Q}_{q}^{\downarrow}$ the sequence $\left(Y_{1}, Y_{2}, \ldots\right)$ is a Markov chain with the same transition kernel and with initial law

$$
\mathbf{Q}_{q}^{\downarrow}\left(Y_{1} \in d y\right)=\check{H}_{q}(d y) .
$$


Proof. The following identities hold:

$$
\begin{aligned}
& \mathbf{Q}_{q}^{\uparrow}\left(Z_{1} \in d y\right)=H_{q}(d y), \\
& \mathbf{Q}_{q}^{\uparrow}\left(Z_{2} \in d y \mid Z_{1}=x\right)=\mathbf{Q}_{q}^{\uparrow}\left(\min \left[X_{\sigma+t}-M ; t \geq 0\right] \in d y \mid M=x\right) \\
& \quad=\mathbf{Q}_{q}^{\downarrow}(m \in d y \mid-m \leq x)=\frac{\check{H}_{q}(-d y)}{\check{H}_{q}(x)} 1_{y \in[-x, 0]} .
\end{aligned}
$$

The third identity follows from Lemma 8.1 the others follow from the definitions of the random variables $Z_{1}$ and $Z_{2}$.

The same arguments show that

$$
\begin{aligned}
& \mathbf{Q}_{q}^{\downarrow}\left(Y_{1} \in d y\right)=\check{H}_{q}(d y), \\
& \mathbf{Q}_{q}^{\downarrow}\left(Y_{2} \in d y \mid Y_{1}=x\right)=\frac{H_{q}(d y)}{H_{q}\left(x^{-}\right)} 1_{y \in[0, x[} .
\end{aligned}
$$

A straightforward induction establishes the rest of the lemma.

Write

$$
\begin{aligned}
U_{q}(d y) & :=\sum_{1}^{+\infty} \mathbf{Q}_{q}^{\uparrow}\left(Z_{n} \in d y\right), & V_{q}(d y) & :=\sum_{1}^{+\infty} \mathbf{Q}_{q}^{\downarrow}\left(Y_{n} \in d y\right), \\
\tau_{x} & :=\sup \left\{n ; Z_{n} \notin[-x, x[\}\right. & & \left(\tau_{x}:=0 \text { if } Z_{1}<x\right), \\
\nu_{x} & :=\sup \left\{n ; Y_{n} \notin[-x, x[\}\right. & & \left(\nu_{x}:=0 \text { if } Y_{1} \geq-x\right) .
\end{aligned}
$$

Lemma 9.2. 1) Under $\mathbf{Q}_{q}^{\uparrow}\left(d w ; \tau_{x}>0\right)$, the sequence $\left(Z_{\tau_{x}-n}\right)_{0 \leq n<\tau_{x}}$ is a subMarkov chain with values in $\mathbf{R}^{*}$ and with initial law

$$
\mathbf{Q}_{q}^{\uparrow}\left(Z_{\tau_{x}} \in d y ; \tau_{x}>0\right)=\left[1 _ { y \in ] - \infty , - x [ } \left[\frac{H_{q}\left(x^{-}\right)}{H_{q}\left((-y)^{-}\right)}+1_{y \in[x,+\infty[}\left[\frac{\check{H}_{q}(x)}{\check{H}_{q}(y)}\right] U_{q}(d y) .\right.\right.
$$

Its transition kernel $R_{q}$ does not depend on $x$ and satisfies the equation

$$
U_{q}(d z) P_{q}(z, d y)=R_{q}(y, d z) U_{q}(d y) \quad \text { on } \mathbf{R}^{*} \times \mathbf{R}^{*} .
$$

2) Under $\mathbf{Q}_{q}^{\downarrow}\left(d w ; \nu_{x}>0\right)$, the sequence $\left(Y_{\nu_{x}-n}\right)_{0 \leq n<\nu_{x}}$ is a sub-Markov chain with values in $\mathbf{R}^{*}$ and with initial law

$$
\mathbf{Q}_{q}^{\downarrow}\left(Y_{\nu_{x}} \in d y, \nu_{x}>0\right)=\left[1_{y \in]-\infty,-x[} \frac{H_{q}\left(x^{-}\right)}{H_{q}\left((-y)^{-}\right)}+1_{y \in[x,+\infty[} \frac{\check{H}_{q}(x)}{\check{H}_{q}(y)}\right] V_{q}(d y) .
$$

Its transition kernel $S_{q}$ does not depend on $x$ and satisfies the equation

$$
V_{q}(d z) P_{q}(z, d y)=S_{q}(y, d z) V_{q}(d y) \quad \text { on } \mathbf{R}^{*} \times \mathbf{R}^{*} .
$$

Proof. It is a standard fact from the theory of time reversal of Markov chains (or processes) that the time $\tau_{x}$ is a so-called "return time" of the Markov chain $\left(Z_{n}\right)$ and consequently that $\left(Z_{\tau_{x}-n}\right)_{0 \leq n<\tau_{x}}$ is a sub-Markov chain with a transition kernel related to that of $\left(Z_{n}\right)$ by the so-called "duality identity"

$$
U_{q}(d z) P_{q}(z, d y)=R_{q}(y, d z) U_{q}(d y) \quad \text { on } \mathbf{R}^{*} \times \mathbf{R}^{*} .
$$

In particular, this transition kernel does not depend on the particular return time $\tau_{x}$. Since we lack an adequate reference, we check this property in our particular case. 
For every $y \in \mathbf{R}^{*}$, let $z \mapsto p_{q}(y, z)$ be a density of the measure $P_{q}(y, d z)$ relative to $U_{q}(d z)$. Then for every integer $m$ (measures involved here are on $\mathbf{R}^{*}$ )

$$
\begin{aligned}
& \mathbf{Q}_{q}^{\uparrow}\left(Z_{\tau_{x}-m} \in d y_{m}, Z_{\tau_{x}-(m-1)} \in d y_{m-1}, \ldots, Z_{\tau_{x}} \in d y_{0}\right) \\
& =\sum_{n=m+1}^{+\infty} \mathbf{Q}_{q}^{\uparrow}\left(Z_{n-m} \in d y_{m}, Z_{n-(m-1)} \in d y_{m-1}, Z_{n-(m-2)} \in d y_{m-2}, \ldots,\right. \\
& \left.Z_{n} \in d y_{0}, \tau_{x}=n\right) \\
& =\sum_{n=m+1}^{+\infty} \mathbf{Q}_{q}^{\uparrow}\left(Z_{n-m} \in d y_{m}, Z_{n-(m-1)} \in d y_{m-1}, Z_{n-(m-2)} \in d y_{m-2}, \ldots,\right. \\
& Z_{n} \in d y_{0}, Z_{n+1} \in\left[-x, x[) 1_{y_{0} \notin[-x, x[}\right. \\
& =\sum_{n=m+1}^{+\infty} \mathbf{Q}_{q}^{\uparrow}\left(Z_{n-m} \in d y_{m}\right) p_{q}\left(y_{m}, y_{m-1}\right) U_{q}\left(d y_{m-1}\right) P_{q}\left(y_{m-1}, d y_{m-2}\right) \ldots
\end{aligned}
$$

$$
P_{q}\left(y_{1}, d y_{0}\right) 1_{y_{0} \notin[-x, x[} P_{q}\left(y_{0},[-x, x[)\right.
$$

$$
=U_{q}\left(d y_{m}\right) p_{q}\left(y_{m}, y_{m-1}\right) U_{q}\left(d y_{m-1}\right) P_{q}\left(y_{m-1}, d y_{m-2}\right) \ldots P_{q}\left(y_{1}, d y_{0}\right) 1_{y_{0} \notin[-x, x[} .
$$

The second identity follows from the definition of our particular time $\tau_{x}$ and from the fact that the sequence $\left|Z_{n}\right|$ is nonincreasing; the other identities are trivial.

We deduce from identity (9.3) that

$$
\mathbf{Q}_{q}^{\uparrow}\left(Z_{\tau_{x}-m} \in d y_{m} \mid Z_{\tau_{x}-(m-1)}, \ldots, Z_{\tau_{x}}\right)=U_{q}\left(d y_{m}\right) p_{q}\left(y_{m}, Z_{\tau_{x}-(m-1)}\right) .
$$

Thus the sequence $\left(Z_{\tau_{x}-n}\right)_{0 \leq n<\tau_{x}}$ is a sub-Markov chain and its transition kernel is $R_{q}(z, d y)=p_{q}(y, z) U_{q}(d y)$. Multiplying the last identity by the measure $U_{q}(d z)$, one recognizes the duality equation.

We now compute the law of $Z_{\tau_{x}}$. Put $m=0$ in the identity (9.2). This yields

$$
\begin{array}{r}
\mathbf{Q}_{q}^{\uparrow}\left(Z_{\tau_{x}} \in d y_{0}\right)=1_{y_{0} \notin[-x, x[} \sum_{n=1}^{+\infty} \mathbf{Q}_{q}^{\uparrow}\left(Z_{n} \in d y_{0}\right) P_{q}\left(y_{0},[-x, x[)\right. \\
=\left(1_{y_{0} \in[x,+\infty[} \frac{\check{H}_{q}(x)}{\check{H}_{q}\left(y_{0}\right)}+1_{\left.y_{0} \in\right]-\infty,-x[} \frac{H_{q}(x)}{H_{q}\left((-y)_{0}^{-}\right)}\right) U_{q}\left(d y_{0}\right) .
\end{array}
$$

The last identity comes from the value of $P_{q}$ given in Lemma 9.1

The corresponding results for the sequence $\left(Y_{\nu_{x}-n}\right)_{0 \leq n<\nu_{x}}$ are derived similarly.

Write

$$
\begin{aligned}
& c_{q}(x, \lambda):=\frac{1}{\check{H}_{q}(x)} \mathbf{Q}_{q}^{\uparrow}\left(e^{-\lambda \sum_{n=1}^{\tau_{x}} Z_{n}} ; \tau_{x} \text { is odd }\right)=\frac{1}{\check{H}_{q}(x)} \mathbf{Q}_{q}^{\uparrow}\left(e^{-\lambda M_{\tau_{x}}} ; \tau_{x} \text { is odd }\right), \\
& b_{q}(x, \lambda):=\frac{1}{H_{q}\left(x^{-}\right)} \mathbf{Q}_{q}^{\uparrow}\left(e^{-\lambda \sum_{n=1}^{\tau_{x}} Z_{n}} ; \tau_{x} \text { is even }\right)=\frac{1}{H_{q}\left(x^{-}\right)} \mathbf{Q}_{q}^{\uparrow}\left(e^{-\lambda m_{\tau_{x}}} ; \tau_{x} \text { is even }\right), \\
& \check{c}_{q}(x, \lambda):=\frac{1}{H_{q}\left(x^{-}\right)} \mathbf{Q}_{q}^{\downarrow}\left(e^{-\lambda \sum_{n=1}^{\nu_{x}} Y_{n}} ; \nu_{x} \text { is odd }\right)=\frac{1}{H_{q}\left(x^{-}\right)} \mathbf{Q}_{q}^{\downarrow}\left(e^{-\lambda m_{\nu_{x}}} ; \nu_{x} \text { is odd }\right), \\
& \check{b}_{q}(x, \lambda):=\frac{1}{\check{H}_{q}(x)} Q_{q}^{\downarrow}\left(e^{-\lambda \sum_{n=1}^{\nu_{x}} Y_{n}} ; \nu_{x} \text { is even }\right)=\frac{1}{\check{H}_{q}(x)} \mathbf{Q}_{q}^{\downarrow}\left(e^{-\lambda M_{\nu_{x}}} ; \nu_{x} \text { is even }\right) .
\end{aligned}
$$


Lemma 9.3. For $\Re(\lambda)>0$ (and $\Re(\lambda)=0$ if $q>0$ or $\lim X_{t}=-\infty \mathbf{P}$-a.s.),

$$
\left|c_{q}(x, \lambda)\right|<+\infty \text { and }\left|b_{q}(x, \lambda)\right|<+\infty,
$$

and

$$
A_{q}(x-, \lambda) b_{q}(x, \lambda)+\check{A}_{q}(x, \lambda) c_{q}(x, \lambda)=\frac{1}{\psi_{q}(\lambda)} .
$$

For $\Re(\lambda)<0$ (and for $\Re(\lambda)=0$ if $q>0$ or $\lim X_{t}=+\infty \mathbf{P}$-a.s.),

$$
\left|\check{c}_{q}(x, \lambda)\right|<+\infty \text { and }\left|\check{b}_{q}(x, \lambda)\right|<+\infty \text {, }
$$

and

$$
A_{q}(x-, \lambda) \check{b}_{q}(x, \lambda)+\check{A}_{q}(x, \lambda) \check{c}_{q}(x, \lambda)=\frac{1}{\check{\psi}_{q}(\lambda)} .
$$

Proof. Write $S_{x}:=\inf \left\{n \mid Z_{n} \in\left[-x, x[\}\right.\right.$. Clearly $S_{x}=\tau_{x}+1$. Moreover,

$$
\begin{gathered}
\mathbf{Q}_{q}^{\uparrow}\left(\tau_{x}=0, Z_{S_{x}} \in d y\right)+\sum_{n=1}^{+\infty} \mathbf{Q}_{q}^{\uparrow}\left(Z_{1} \in d x_{1} \ldots Z_{n} \in d x_{n} \tau_{x}=n, Z_{S_{x}} \in d y\right) \\
=\mathbf{Q}_{q}^{\uparrow}\left(\tau_{x}=0, Z_{S_{x}} \in d y\right)+\sum_{n=1}^{+\infty} 1_{x_{n} \notin[-x, x[} 1_{y \in[-x, x}\left[\mathbf { Q } _ { q } ^ { \uparrow } \left(Z_{1} \in d x_{1}, \ldots,\right.\right. \\
\left.Z_{n} \in d x_{n}, Z_{n+1} \in d y\right) \\
=\mathbf{Q}_{q}^{\uparrow}\left(Z_{1} \in d y\right) 1_{y \in[-x, x[}+\sum_{n=1}^{+\infty} 1_{x_{n} \notin[-x, x[} 1_{y \in[-x, x}\left[\mathbf { Q } _ { q } ^ { \uparrow } \left(Z_{1} \in d x_{1}, \ldots,\right.\right. \\
=H_{q}(d y) 1_{y \in[0, x[}\left[1+\sum_{n \text { is even }, n \geq 2} 1_{\left.x_{n} \in\right]-\infty,-x[} \mathbf{Q}_{q}^{\uparrow}\left(Z_{1} \in d x_{n}\right) P_{q}\left(x_{n}, d y\right)\right. \\
\left.\left.Z_{n} \in d x_{n}\right) \frac{1}{H_{q}\left(\left(-x_{n}\right)^{-}\right)}\right] \\
+\check{H}_{q}(-d y) 1_{y \in[-x, 0]}\left[\sum _ { n \text { is odd } } 1 _ { x _ { n } \in [ x , + \infty [ } \mathbf { Q } _ { q } ^ { \uparrow } \left(Z_{1} \in d x_{1}, \ldots,\right.\right. \\
\left.\left.Z_{n} \in d x_{n}\right) \frac{1}{\check{H}_{q}\left(x_{n}\right)}\right] .
\end{gathered}
$$

Again, the first identity is a consequence of the fact that the sequence $\left|Z_{n}\right|$ is nonincreasing together with the definition of times $\tau_{x}$ and $S_{x}$. The third identity follows from Lemma 9.1 .

Now, let us check that the $\sigma$-field $\sigma\left(Z_{n} 1_{n \leq \tau_{x}} ; n \geq 1\right)$ is $\sigma$-finite. To see this, first note that the variable $Z_{1} 1_{\tau_{x} \neq 0}+1_{\tau_{x}=0}$ is positive and is $\sigma\left(Z_{n} 1_{n \leq \tau_{x}} ; n \geq 1\right)$ measurable. The variable $Z_{1} 1_{\tau_{x} \neq 0}$ has law $H_{q}(d y) 1_{y \in[x,+\infty}$, which is $\sigma$-finite, and the event $\left\{\tau_{x}=0\right\}$ has measure $H_{q}\left(x^{-}\right)$. So one can deduce the conditional law of the variable $Z_{T_{x}}$ on $\sigma\left(Z_{n} 1_{n \leq \tau_{x}} ; n \geq 1\right)$ from the preceding identities:

$$
\begin{aligned}
\mathbf{Q}_{q}^{\uparrow}\left(Z_{S_{x}} \in d y \mid Z_{n} 1_{n \leq \tau_{x}} ; n \geq 1\right) & =1_{\tau_{x} \text { is odd }} \frac{\check{H}_{q}(-d y)}{\check{H}_{q}(x)} 1_{y \in[-x, 0]} \\
& +1_{\tau_{x} \text { is even }} \frac{H_{q}(d y)}{H_{q}\left(x^{-}\right)} 1_{y \in[0, x[} .
\end{aligned}
$$


Moreover,

$$
\begin{aligned}
A_{q}\left(x^{-}, \lambda\right)=\mathbf{Q}_{q}^{\uparrow}\left(e^{-\lambda F} ; M<x\right) & =\mathbf{Q}_{q}^{\uparrow}\left(e^{-\lambda \sum_{1}^{+\infty} Z_{n}} ; Z_{1}<x\right) \\
& =\int_{[0, x[} \mathbf{Q}_{q}^{\uparrow}\left(e^{-\lambda \sum_{1}^{+\infty} Z_{n}} \mid Z_{1}=y\right) H_{q}(d y) .
\end{aligned}
$$

Similarly,

$$
\check{A}_{q}(x, \lambda)=\int_{[-x, 0]} \mathbf{Q}_{q}^{\downarrow}\left(e^{-\lambda \sum_{1}^{+\infty} Y_{n}} \mid Y_{1}=y\right) \check{H}_{q}(-d y) .
$$

Remember that

The transition kernels of $\left(Z_{n}\right)$ under $\mathbf{Q}_{q}^{\uparrow}$ and of $\left(Y_{n}\right)$ under $\mathbf{Q}_{q}^{\downarrow}$ are the same.

$S_{x}$ is a stopping time of the chain $\left(Z_{n}\right)$

$$
\text { and } \sigma\left(Z_{n} 1_{n \leq S_{x}} ; n \geq 1\right) \supset \sigma\left(Z_{n} 1_{n \leq \tau_{x}} ; n \geq 1\right) .
$$

We deduce from properties (9.4), (9.5), (9.6), (9.7), (9.8), the following identity:

$$
\mathbf{Q}_{q}^{\uparrow}\left(e^{-\lambda \sum_{S_{x}}^{+\infty} Z_{n}} \mid Z_{n} 1_{n \leq \tau_{x}} ; n \geq 1\right)=1_{\tau_{x} \text { is odd }} \frac{\check{A}_{q}(x, \lambda)}{\check{H}_{q}(x)}+1_{\tau_{x} \text { is even }} \frac{A_{q}\left(x^{-}, \lambda\right)}{H_{q}\left(x^{-}\right)} .
$$

Therefore,

$$
\begin{aligned}
& \frac{1}{\psi_{q}(\lambda)}=\mathbf{Q}_{q}^{\uparrow}\left(e^{-\lambda F}\right)=\mathbf{Q}_{q}^{\uparrow}\left(e^{-\lambda \sum_{1}^{+\infty} Z_{n}}\right)=\mathbf{Q}_{q}^{\uparrow}\left(e^{-\lambda \sum_{1}^{\tau_{x}} Z_{n}} e^{-\lambda \sum_{S_{x}}^{+\infty} Z_{n}}\right) \\
& \quad=\mathbf{Q}_{q}^{\uparrow}\left(e^{-\lambda \sum_{1}^{\tau_{x}} Z_{n}} 1_{\tau_{x} \text { is even }}\right) \frac{A_{q}\left(x^{-}, \lambda\right)}{H_{q}\left(x^{-}\right)}+\mathbf{Q}_{q}^{\uparrow}\left(e^{-\lambda \sum_{1}^{\tau_{x}} Z_{n}} 1_{\tau_{x} \text { is odd }}\right) \frac{\check{A}_{q}(x, \lambda)}{\check{H}_{q}(x)} \\
& \quad=b_{q}(x, \lambda) A_{q}\left(x^{-}, \lambda\right)+c_{q}(x, \lambda) \check{A}_{q}(x, \lambda) .
\end{aligned}
$$

The condition that $c_{q}(x, \lambda)$ and $b_{q}(x, \lambda)$ be finite has to be checked only in the case $q=0$ and $\Re(\lambda)>0$. It is enough to check this fact for positive real $\lambda$. In this case the real numbers $A_{0}\left(x^{-}, \lambda\right), \check{A}_{0}(x, \lambda)$ are positive and $\frac{1}{\psi_{0}(\lambda)}$ is finite. Thus the previous identity shows that the (positive but possibly infinite a priori) $c_{0}(x, \lambda)$ and $b_{0}(x, \lambda)$ are actually finite.

The second part of the lemma is proved similarly.

Lemma 9.4. For $\Re(\lambda)>0$ (and for $\Re(\lambda)=0$ if $q>0$ or if $\lim X_{t}=-\infty \mathbf{P}$-a.s.),

$$
\begin{aligned}
& c_{q}(x, \lambda)=\int_{[x,+\infty[} e^{-\lambda y} b_{q}(y, \lambda) \frac{H_{q}(d y)}{\check{H}_{q}(y)}, \\
& b_{q}(x, \lambda)=1+\int_{] x,+\infty[} e^{\lambda y} c_{q}(y, \lambda) \frac{\check{H}_{q}(d y)}{H_{q}\left(y^{-}\right)} .
\end{aligned}
$$

For $\Re(\lambda)<0$ (and for $\Re(\lambda)=0$ if $q>0$ or $\lim X_{t}=+\infty \mathbf{P}$-a.s.),

$$
\begin{aligned}
& \check{c}_{q}(x, \lambda)=\int_{] x,+\infty[} e^{\lambda y} \check{b}_{q}(y, \lambda) \frac{\check{H}_{q}(d y)}{H_{q}\left(y^{-}\right)}, \\
& \check{b}_{q}(x, \lambda)=1+\int_{[x,+\infty[} e^{-\lambda y} \check{c}_{q}(y, \lambda) \frac{H_{q}(d y)}{\check{H}_{q}(y)} .
\end{aligned}
$$


Proof. We first extend the transition kernel of $\left(Z_{n}\right), R_{q}$, by setting $R_{q}(y,\{-\infty\}):=$ $1-R_{q}\left(y, \mathbf{R}^{*}\right)$ for $y \neq 0$. Integrate with respect to $z$ the "duality equation", $1_{y z \neq 0} U_{q}(d z) P_{q}(z, d y)=1_{y z \neq 0} U_{q}(d y) R_{q}(y, d z)$. This yields

$$
\begin{aligned}
1_{y \in \mathbf{R}^{*}} R_{q}\left(y, \mathbf{R}^{*}\right) U_{q}(d y) & =1_{y \in \mathbf{R}^{*}} \int_{\mathbf{R}^{*}} U_{q}(d z) P_{q}(z, d y) \\
& =1_{y \in \mathbf{R}^{*}} \sum_{2}^{+\infty} Q_{q}^{\uparrow}\left(Z_{n} \in d y\right)=1_{y \in \mathbf{R}^{*}}\left(U_{q}(d y)-H_{q}(d y)\right) .
\end{aligned}
$$

From this it follows that

$$
R_{q}(y,\{-\infty\}) U_{q}(d y)=H_{q}(d y) \text { for } y \neq 0 .
$$

For $y \neq 0$, denote by $p^{y}$ the distribution of the Markov chain starting from $y$ and with transition kernel $R_{q},\left(S_{n}\right)$ the canonical process of the space $\left(\mathbf{R}^{*} \cup\{-\infty\}\right)^{\mathbf{N}}$, where $-\infty$ is the cemetery point and $\xi$ the lifetime of the process $\left.S_{n}\right)$. From the distribution of $Z_{\tau_{x}}$ as stated in Lemma 9.2, it follows that

$$
c_{q}(x, \lambda)=\frac{1}{\check{H}_{q}(x)} \mathbf{Q}_{q}^{\uparrow}\left(e^{-\lambda \sum_{n=1}^{\tau_{x}} Z_{n}} ; \tau_{x} \text { is odd }\right)=\int_{[x,+\infty[} p^{y}\left(e^{-\lambda \sum_{1}^{\xi} S_{n}}\right) \frac{U_{q}(d y)}{\check{H}_{q}(y)}
$$

and

$$
\begin{aligned}
& b_{q}(x, \lambda)=\frac{1}{H_{q}\left(x^{-}\right)} \mathbf{Q}_{q}^{\uparrow}\left(e^{-\lambda \sum_{n=1}^{\tau_{x}} Z_{n}} ; \tau_{x} \text { is even }\right) \\
& \quad=\frac{1}{H_{q}\left(x^{-}\right)}\left[\mathbf{Q}_{q}^{\uparrow}\left(\tau_{x}=0\right)+\int_{]-\infty,-x]} p^{y}\left(e^{-\lambda \sum_{1}^{\xi} S_{n}}\right) \cdot U_{q}(d y) \frac{H_{q}\left(x^{-}\right)}{H_{q}\left((-y)^{-}\right)}\right] \\
& \quad=1+\int_{]-\infty,-x[} p^{y}\left(e^{-\lambda \sum_{1}^{\xi} S_{n}}\right) \cdot \frac{U_{q}(d y)}{H_{q}\left((-y)^{-}\right)} .
\end{aligned}
$$

On the other hand, for every $y \neq 0$,

$$
\begin{aligned}
& p^{y}\left(e^{-\lambda \sum_{1}^{\xi} S_{n}}\right) U_{q}(d y)=e^{-\lambda y}\left[\int_{[-\infty,+\infty[\backslash\{0\}} p^{z}\left(e^{-\lambda \sum_{1}^{\xi} S_{n}}\right) R_{q}(y, d z)\right] U_{q}(d y) \\
& \quad=e^{-\lambda y}\left[R_{q}(y,\{-\infty\}) U_{q}(d y)+\int_{]-\infty,+\infty[\backslash\{0\}} p^{z}\left(e^{-\lambda \sum_{1}^{\xi} S_{n}}\right) U_{q}(d z) P_{q}(z, d y)\right] \\
& \quad=e^{-\lambda y}\left[1+\int_{]-\infty,-y[} p^{z}\left(e^{-\lambda \sum_{1}^{\xi} S_{n}}\right) \frac{U_{q}(d z)}{H_{q}(-z)}\right] H_{q}(d y)=e^{-\lambda y} b_{q}(y, \lambda) H_{q}(d y) .
\end{aligned}
$$

The first identity follows from the fact that $\left(S_{n}\right)$ has the Markov property under $p^{y}$ with transition kernel $R_{q}$. The duality equation (9.1) proves the second identity. Lemma 9.1, together with the identity (9.9), impies the third identity. The fourth identity derives from (9.11).

Using the preceding identity, we can derive from (9.10) that

$$
c_{q}(x, \lambda)=\int_{] x,+\infty[} e^{-\lambda y} b_{q}(y, \lambda) \frac{H_{q}(d y)}{\check{H}_{q}(y)} .
$$

The other assertions of the lemma are proved in a similar way.

Lemma 9.5. For every complex number $\lambda$ with $\Re(\lambda)>0$ (and $\Re(\lambda)=0$ if $q>0$ or $\lim _{t \rightarrow+\infty} X_{t}=-\infty, \mathbf{P}$-ps),

$$
\psi_{q}(\lambda) c_{q}(x, \lambda)=C_{q}\left(x^{-}, \lambda\right) \quad \text { and } \quad \psi_{q}(\lambda) b_{q}(x, \lambda)=B_{q}(x, \lambda)
$$


For every complex number $\lambda$ with $\Re(\lambda)<0$ (and $\Re(\lambda)=0$ if $q>0$ or $\lim _{t \rightarrow+\infty} X_{t}=$ $+\infty, \mathbf{P}-p s)$

$$
\check{\psi}_{q}(\lambda) \check{c}_{q}(x, \lambda)=\check{C}_{q}(x, \lambda) \quad \text { and } \quad \check{\psi}_{q}(\lambda) \check{b}_{q}(x, \lambda)=\check{B}_{q}\left(x^{-}, \lambda\right) .
$$

Proof. Recall the definition (6) of the time $U^{x}$,

$$
U^{x}=\inf \left\{t \mid S_{t}-I_{t} \geq x \text { and } S_{t}=X_{t}\right\} \wedge \inf \left\{t \mid S_{t}-I_{t}>x \text { and } X_{t}=I_{t}\right\} .
$$

Let $B^{x}$ be the event $B^{x}:=\left\{X_{U^{x}}=S_{U^{x}}, U^{x}<+\infty\right\}$ and denote by $\hat{X}$ the process $\hat{X}_{t}(w)=F-X_{t-}(w)$. Add a ${ }^{\wedge}$ for the corresponding objects. Denote by $X^{\uparrow}$ and $X^{\downarrow}$ the processes $\left(X_{t+\rho}-m ; t \geq 0\right\}$ and $\left(X_{t+\sigma}-M ; t \geq 0\right\}$, respectively.

A quick look at the picture will convince the reader that the following two properties hold:

$$
\left\{\tau_{x}\left(X^{\uparrow}\right) \text { is odd }\right\}=\hat{B}^{x}, \quad \sum_{1}^{\tau_{x}\left(X^{\uparrow}\right)} Z_{n}\left(X^{\uparrow}\right)=\hat{M}-\hat{I}_{\hat{U}_{x}} \quad \text { on } \hat{B}^{x} .
$$

Pick an event $A$ such that $0<\mathbf{Q}_{q}^{\downarrow}(A)<+\infty$ and get

$$
\begin{aligned}
\mathbf{Q}_{q}^{\downarrow} & (A) \check{H}_{q}(x) c_{q}(x, \lambda)=\mathbf{Q}_{q}^{\downarrow}(A) \mathbf{Q}_{q}^{\uparrow}\left(e^{-\lambda \sum_{n=1}^{\tau_{x}} Z_{n}} ; \tau_{x} \text { is odd }\right) \\
& =\mathbf{Q}_{q}\left(A\left(m-X_{(\rho-t)^{-}} ; t \geq 0\right) ; e^{-\lambda \sum_{n=1}^{\tau_{x}\left(X^{\uparrow}\right)} Z_{n}} ; \tau_{x}\left(X^{\uparrow}\right) \text { is odd }\right) \\
& =\mathbf{Q}_{q}\left(A\left(\hat{X}^{\downarrow}\right), e^{-\lambda\left(\hat{M}-\hat{I}_{\hat{U}_{x}}\right)} ; \hat{B}_{x}\right) \\
& =\mathbf{Q}_{q}\left(e^{-\lambda\left(M-I_{\left.U^{x}\right)}\right.} ; B^{x}, A\left(X^{\downarrow}\right)\right) .
\end{aligned}
$$

The first identity follows from the definition of $c_{q}$, the second one from Proposition 7.1 the third one from (9.12) and the fourth one from the identity in law of $\hat{X}$ and $X$ under $\mathbf{Q}_{q}$.

On the other hand, $U^{x}$ is a stopping time and it is smaller than $\sigma$ on the event $B^{x}$. Therefore we get (denote by $\theta_{U^{x}}^{\circ}$ the path $\left(X_{t+U^{x}}-X_{U^{x}} ; t \geq 0\right)$ )

$$
M-I_{U^{x}}=\left(X_{U^{x}}-I_{U^{x}}\right)+M \circ \theta_{U^{x}}^{\circ} \text { and } \quad A\left(X^{\downarrow}\right)=A\left(X^{\downarrow} \circ \theta_{U_{x}}^{\circ}\right) \text { on } B^{x} \text {. }
$$

From this, it follows that

$$
\begin{aligned}
& \mathbf{Q}_{q}\left(e^{-\lambda\left(M-I_{U^{x}}\right)} ; B^{x} ; A\left(X^{\downarrow}\right)\right)=\mathbf{Q}_{q}\left(e^{-\lambda\left(X_{U^{x}}-I_{U^{x}}\right)} ; B^{x} ; e^{-\lambda M \circ \theta_{U_{x}}^{\circ}} ; A\left(X^{\downarrow} \circ \theta_{U^{x}}^{\circ}\right)\right) \\
& \quad=\mathbf{P}_{q}\left(e^{-\lambda\left(X_{U^{x}}-I_{U^{x}}\right)} ; B^{x}\right) \mathbf{Q}_{q}\left(e^{-\lambda M} ; A\left(X^{\downarrow}\right)\right) .
\end{aligned}
$$

The first identity follows from (9.14), and the second one derives from the Markov property at time $U^{x}$ as follows:

The first identity in Proposition 6.3 for $\lambda=0, \mu_{1}=\lambda$ and $q_{1}=q_{2}=q$ implies

$$
\mathbf{P}_{q}\left(e^{-\lambda\left(X_{U^{x}-I_{U} x}\right.} ; B^{x}\right)=\check{H}_{q}(x) C_{q}\left(x^{-}, \lambda\right) .
$$

Finally, we obtain from Proposition 7.1

$$
\mathbf{Q}_{q}\left(e^{-\lambda M} ; A\left(X^{\downarrow}\right)\right)=\mathbf{Q}_{q}^{\uparrow}\left(e^{-\lambda F}\right) \mathbf{Q}_{q}^{\downarrow}(A)=\frac{1}{\psi_{q}(\lambda)} \mathbf{Q}_{q}^{\downarrow}(A) .
$$

Putting together equations (9.13), (9.15), (9.16), (9.17), we obtain

$$
\begin{aligned}
& \mathbf{Q}_{q}^{\downarrow}(A) \check{H}_{q}(x) c_{q}(x, \lambda)=\mathbf{Q}_{q}\left(e^{-\lambda\left(M-I_{U_{x}}\right)} ; B_{x}, A\left(X^{\downarrow}\right)\right) \\
& \quad=\mathbf{P}_{q}\left(e^{-\lambda\left(X_{U_{x}}-I_{U_{x}}\right)} ; B^{x}\right) \mathbf{Q}_{q}\left(e^{-\lambda M} ; A\left(X^{\downarrow}\right)\right)=\check{H}_{q}(x) C_{q}\left(x^{-}, \lambda\right) \frac{1}{\psi_{q}(\lambda)} \mathbf{Q}_{q}^{\downarrow}(A) .
\end{aligned}
$$


Multiplying these equations by $\frac{\psi_{q}(\lambda)}{\mathbf{Q}_{q}^{\downarrow}(A) \check{H}_{q}(x)}$, this yields

$$
C_{q}\left(x^{-}, \lambda\right)=\psi_{q}(\lambda) c_{q}(x, \lambda) .
$$

We now turn to the proof of the second identity of the lemma. To this end, recall that $V_{x}=\inf \left\{t \mid X_{t}-S_{t}<-x\right\}$.

Notice further that if $U^{x}=+\infty$ or $I_{U^{x}}=X_{U^{x}}$, then $V_{x}=U^{x}$. Moreover (denoting by $\theta_{U^{x}}^{\circ}$ the path $\left(X_{s+U^{x}}-X_{U^{x}} ; s \geq 0\right)$ ), if $S_{U^{x}}=X_{U^{x}}$, then $V_{x}=U^{x}=$ $U^{x}+V_{x} \circ \theta_{U^{x}}^{\circ}, L\left(\omega, d t+U^{x}\right) 1_{t \geq 0}=L\left(\theta_{U^{x}}^{\circ}(w), d t\right)$ and $S_{U^{x}+t}=X_{U^{x}}+S_{t} \circ \theta_{U^{x}}^{\circ}$.

Therefore, we deduce the identity of random variables,

$$
\begin{aligned}
& \int_{\left[0, V_{x}[\right.} e^{-\lambda S_{t}} L(d t) \\
& \quad=\int_{\left[0, U^{x}[\right.} e^{-\lambda S_{t}} L(d t)+1_{\left\{X_{U^{x}}=S_{U^{x}} ; U^{x}<+\infty\right\}} e^{-\lambda X_{U^{x}}}\left[\int_{\left[0, V_{x}[\right.} e^{-\lambda S_{t}} L(d t)\right] \circ \theta_{U_{x}}^{\circ} .
\end{aligned}
$$

Thus, by using the Markov property of $X$ at time $U^{x}$, we obtain

$$
\begin{aligned}
& \mathbf{P}_{q} \int_{\left[0, V_{x}[\right.} e^{-\lambda S_{t}} L(d t)=\mathbf{P}_{q}\left(\int_{\left[0, U^{x}[\right.} e^{-\lambda S_{t}} L(d t)\right) \\
& \quad+\mathbf{P}_{q}\left(e^{-\lambda X_{U^{x}}} ; X_{U^{x}}=S_{U^{x}} ; U^{x}<+\infty\right) \mathbf{P}_{q}\left(\left[\int_{\left[0, V_{x}[\right.} e^{-\lambda S_{t}} L(d t)\right]\right) .
\end{aligned}
$$

Notice that

$$
\frac{1}{B_{q}(x, \lambda)}=\mathbf{P}_{q}\left(\int_{\left[0, V_{x}[\right.} e^{-\lambda S_{t}} L(d t)\right)
$$

follows from (3.1). Let us now check that

$$
A_{q}\left(x^{-}, \lambda\right)=\mathbf{P}_{q}\left(\int_{\left[0, U^{x}[\right.} e^{-\lambda S_{t}} L(d t)\right)
$$

Regularizing to the left in the definition of $A_{q}(x, \lambda)$, we obtain

$$
A_{q}\left(x^{-}, \lambda\right)=\mathbf{P}_{q}\left(\int_{\left[0, U^{x-}[\right.} e^{-\lambda S_{t}} L(d t)\right),
$$

where $U^{x-}=\inf \left\{t \mid S_{t}-I_{t} \geq x\right\}$. Notice that if $U^{x-}$ and $U^{x}$ are different, then $I_{U^{x-}}=X_{U^{x-}}$ and $U^{x} \leq \inf \left\{t \geq U^{x-} \mid S_{t}=X_{t}\right\}$. Thus the measure $L(d t)$ puts no mass on the interval $\left[U^{x-}, U^{x}[\right.$ and equation (9.20) holds.

On the other hand, use the first identity of Proposition 6.3 for $\mu_{1}=\lambda$ and $q_{1}=q_{2}=q$ to obtain

$$
\mathbf{P}_{q}\left(e^{-\lambda X_{U^{x}}} ; X_{U^{x}}=S_{U^{x}} ; U^{x}<+\infty\right)=\check{A}_{q}(x, \lambda) C_{q}\left(x^{-}, \lambda\right) .
$$

From equations (9.18), (9.19), (9.20) and (9.21), it follows that

$$
\frac{1}{B_{q}(x, \lambda)}=A_{q}\left(x^{-}, \lambda\right)+\check{A}_{q}(x, \lambda) C_{q}\left(x^{-}, \lambda\right) \frac{1}{B_{q}(x, \lambda)} .
$$

Equivalently,

$$
B_{q}(x, \lambda) A_{q}\left(x^{-}, \lambda\right)+\check{A}_{q}(x, \lambda) C_{q}\left(x^{-}, \lambda\right)=1 .
$$


Upon comparing with the identity obtained in Lemma $9.3: A_{q}\left(x^{-}, \lambda\right) b_{q}(x, \lambda)+$ $\check{A}_{q}(x, \lambda) c_{q}(x, \lambda)=\frac{1}{\psi_{q}(x, \lambda)}$ and the identity already obtained: $C_{q}\left(x^{-}, \lambda\right)=$ $\psi_{q}(x, \lambda) c_{q}(x, \lambda)$, we get the following:

$$
B_{q}(x, \lambda)=\psi_{q}(\lambda) \check{b}_{q}(x, \lambda) .
$$

The other two identities of the lemma are obtained similarly.

Properties 2 and 3 of Theorem 4.1 follow from Lemmas 9.3 , 9.4 and 9.5 .

\section{Proof of Theorem 4.2}

\subsection{Two lemmas.}

Lemma 10.1. For all $x \in] 0,+\infty[$ and $i u \in i \mathbf{R}$,

$$
\begin{aligned}
& B_{q}(x, i u) \check{B}_{q}\left(x^{-}, i u\right)-C_{q}\left(x^{-}, i u\right) \check{C}_{q}(x, i u)=\phi(i u)+q, \\
& \check{B}_{q}(x, i u)=(\phi(i u)+q) A_{q}(x, i u)+C_{q}(x, i u), \\
& B_{q}(x, i u)=(\phi(i u)+q) \check{A}_{q}(x, i u)+\check{C}_{q}(x, i u) .
\end{aligned}
$$

Comments. According to Theorem 4.1, the three pairs of functions $\left(A_{q}\left(x^{-}, i u\right)\right.$, $\left.\check{A}_{q}(x, i u)\right),\left(C_{q}\left(x^{-}, i u\right), B_{q}(x, i u)\right)$ and $\left(\check{B}_{q}\left(x^{-}, i u\right), \check{C}_{q}(x, i u)\right)$ satisfy the same differential equation in $x$, in the sense of distribution theory. If the coefficients of this equation were sufficiently regular, the second and the third identities of Lemma10.1 would follow from the fact that the space of solutions of this differential equation is 2-dimensional, and the first identity would follow from the Wronskian identity. In the next proof, we check that these results still hold in our setting by using Stieltjes integral calculus.

Proof. We will denote by $\mu[x+\infty]$ (resp. $\mu] x,+\infty])(x \in] 0,+\infty])$ any function definite for $x$ positive, complex-valued, left (resp. right) continuous and having bounded variations on $[x,+\infty]$ (resp. $] x,+\infty[$ ) for every $x \in] 0,+\infty]$ and $\mu(d x)$ will be the associated complex measure on $] 0,+\infty]$. Similarly, $\mu[0, x]$ (resp. $\mu[0, x[$ ) will denote a complex-valued function, right (resp. left) continuous and having bounded variations on $[0, x]$ (resp. $[0, x[$ ) for every $x \in[0,+\infty[$ and $\mu(d x)$ will be the associated complex measure on $[0,+\infty[$.

We now state a few facts about Stieltjes integral calculus.

An easy application of Fubini's theorem yields, for all positive $x$,

$$
\left.\left.\left.\left.\mu_{1}[x,+\infty] \mu_{2}\right] x,+\infty\right]=\int_{] x,+\infty]} \mu_{1}[y,+\infty] \mu_{2}(d y)+\int_{[x,+\infty[} \mu_{2}\right] y,+\infty\right] \mu_{1}(d y)
$$

If the complex measure $\mu_{1}(d x)$ is also integrable on $[0, x[$, we have

$$
\mu_{1}\left[0, x\left[\mu_{2}\right] x,+\infty\right]=\int_{] x,+\infty]} \mu_{1}\left[0, y\left[\mu_{2}(d y)-\int_{[x,+\infty[} \mu_{2}\right] y,+\infty\right] \mu_{1}(d y) .
$$

[To establish it, add it with the previous one.] When adding the term

$$
\sum_{y \in[x,+\infty[} \mu_{1}(\{y\}) \mu_{2}(\{y\})
$$

in both integrals of the right-hand side, we obtain

$$
\mu_{1}\left[0, x\left[\mu_{2}\right] x,+\infty\right]=\int_{] x,+\infty]} \mu_{1}[0, y] \mu_{2}(d y)-\int_{[x,+\infty[} \mu_{2}[y,+\infty] \mu_{1}(d y)+\mu_{1}(\{x\}) \mu_{2}(\{x\}) .
$$


By regularizing on the right, we obtain

$$
\left.\left.\mu_{1}[0, x] \mu_{2}\right] x,+\infty\right]=\int_{] x,+\infty]} \mu_{1}[0, y] \mu_{2}(d y)-\int_{] x,+\infty[} \mu_{2}[y,+\infty] \mu_{1}(d y) .
$$

Applying the previous equation to $\mu_{1}[0, x]:=\frac{1}{\left.\mu_{2} \mid x,+\infty\right]}$ when $\frac{1}{\left.\left.\mu_{2}\right] x,+\infty\right]}$ has bounded variations on $[0, x]$ for every positive $x$, we get the identity of complex measures

$$
\left.\mu_{1}[0, x]:=\frac{1}{\left.\left.\mu_{2}\right] x,+\infty\right]} \Longrightarrow \mu_{1}[0, y] \mu_{2}(d y)=\mu_{2}[y,+\infty] \mu_{1}(d y) \quad \text { on }\right] 0,+\infty[
$$

and we deduce

$$
\left.\mu_{1}[0, x]=\frac{1}{\left.\left.\mu_{2}\right] x,+\infty\right]} \Longrightarrow \mu_{1}(d y)=\frac{\mu_{2}(d y)}{\left.\left.\mu_{2}\right] y,+\infty\right] \mu_{2}[y,+\infty]} \quad \text { on }\right] 0,+\infty[.
$$

We now go on to proving the first identity of Lemma 10.1. The equations $\check{B}_{q}\left(x^{-}, i u\right)=\check{\psi}_{q}(i u)+\int_{[x,+\infty[} \check{C}_{q}(y, i u) e^{-i u y} \frac{H_{q}(d y)}{\check{H}_{q}(y)}$ and $B_{q}(x, i u)=\psi_{q}(i u)+$ $\int_{] x,+\infty[} C_{q}\left(y^{-}, i u\right) e^{i u y} \frac{\check{H}_{q}(d y)}{H_{q}\left(y^{-}\right)}$of Theorem 4.1$]$ imply that $\check{B}_{q}\left(x^{-}, i u\right)$ and $B_{q}(x, i u)$ are of the form $\mu_{1}[x,+\infty]$ and $\left.\left.\mu_{2}\right] x,+\infty\right]$, respectively. Now applying identity (10.1) to these two functions, we obtain

$$
\begin{aligned}
\check{B}_{q}\left(x^{-}, i u\right) B_{q}(x, i u)=\check{\psi}_{q}(i u) \psi_{q}(i u)+ & \int_{] x,+\infty[} \check{B}\left(y^{-}, i u\right) \cdot C\left(y^{-}, i u\right) e^{i u y} \frac{\check{H}_{q}(d y)}{H_{q}\left(y^{-}\right)} \\
& +\int_{[x,+\infty[} B(y, i u) . \check{C}(y, i u) e^{-i u y} \frac{H_{q}(d y)}{\check{H}_{q}(y)} .
\end{aligned}
$$

Equations $C_{q}\left(x^{-}, i u\right)=\int_{[x,+\infty[} B_{q}(y, i u) e^{-i u y} \frac{H_{q}(d y)}{\check{H}_{q}(y)} \quad$ and $\quad \check{C}_{q}(x, i u)=$ $\int_{] x,+\infty[} \check{B}_{q}\left(y^{-}, i u\right) e^{i u y} \frac{\check{H}_{q}(d y)}{H_{q}\left(y^{-}\right)}$of Theorem 4.1 imply that $C_{q}\left(x^{-}, i u\right)$ and $\check{C}_{q}(x, i u)$ are also of the form $\mu_{1}[x,+\infty]$ and $\left.\left.\mu_{2}\right] x,+\infty\right]$, respectively. Now applying identity (10.1) to these new functions yields

$$
\begin{aligned}
C_{q}\left(x^{-}, i u\right) \check{C}_{q}(x, i u)=\int_{] x,+\infty[} C_{q}\left(y^{-}, i u\right) \cdot \check{B}_{q}\left(y^{-}, i u\right) e^{i u y} \frac{\check{H}_{q}(d y)}{H_{q}\left(y^{-}\right)} \\
\quad+\int_{[x,+\infty[} \check{C}_{q}(y, i u) \cdot B_{q}(y, i u) e^{-i u y} \frac{H_{q}(d y)}{\check{H}_{q}(y)} .
\end{aligned}
$$

Upon substracting equation (10.4) from (10.5), we obtain

$$
\check{B}_{q}\left(x^{-}, i u\right) B_{q}(x, i u)-C_{q}\left(x^{-}, i u\right) \check{C}_{q}(x, i u)=\check{\psi}_{q}(i u) \psi_{q}(i u)=\phi(i u)+q .
$$

Let us now establish the second identity of Lemma 10.1. When multiplying by $\phi(i u)+q$ the identity of Theorem $4.1\left(\check{A}_{q}(x, i u) \check{B}_{q}\left(x^{-}, i u\right)+A_{q}\left(x^{-}, i u\right) \check{C}_{q}(x, i u)=1\right)$ and subtracting it from (10.6), we get

$$
\begin{aligned}
\check{B}_{q}\left(x^{-}, i u\right)\left[B_{q}(x, i u)\right. & \left.-(\phi(i u)+q) \check{A}_{q}(x, i u)\right] \\
& -\check{C}_{q}(x, i u)\left[C_{q}\left(x^{-}, i u\right)+(\phi(i u)+q) A_{q}\left(x^{-}, i u\right)\right]=0 .
\end{aligned}
$$

Recall identity (3.2) $), \frac{1}{\widetilde{B}_{q}(x, i u)}=\mathbf{P}\left(\int_{\left[0, V^{x}[\right.} e^{-i u I_{t}-q t} \check{L}(d t)\right)$. This implies that neither $\check{B}_{q}(x, i u)$ nor $\check{B}_{q}\left(x^{-}, i u\right)$ vanishes. This enables us to multiply equation (10.6) by 
the measure $\frac{1}{\check{B}_{q}\left(x^{-}, i u\right) \check{B}_{q}(x, i u)} e^{-i u x} \frac{H_{q}(d x)}{\check{H}_{q}(x)}$, and obtain

$$
\begin{aligned}
& {\left[\frac{1}{\check{B}_{q}(x, i u)}\right]\left[\left(B_{q}(x, i u)-(\phi(i u)+q) \check{A}_{q}(x, i u)\right) e^{-i u x} \frac{H_{q}(d x)}{\check{H}_{q}(x)}\right]} \\
& \quad-\left[C_{q}\left(x^{-}, i u\right)+(\phi(i u)+q) A_{q}\left(x^{-}, i u\right)\right]\left[\frac{\check{C}_{q}(x, i u)}{\check{B}_{q}\left(x^{-}, i u\right) \check{B}_{q}(x, i u)} e^{-i u x} \frac{H_{q}(d x)}{\check{H}_{q}(x)}\right]=0 .
\end{aligned}
$$

Put $q>0$. We claim that equation (10.7) is of the form $\mu_{1}[0, x] \mu_{2}(d x)-$ $\mu_{2}[x,+\infty] \mu_{1}(d x)=0$, where

$$
\mu_{1}[0, x]:=\frac{1}{\check{B}_{q}(x, i u)} \quad \text { and } \quad \mu_{2}[x,+\infty]:=C_{q}\left(x^{-}, i u\right)+(\phi(i u)+q) A_{q}\left(x^{-}, i u\right) .
$$

To see this, first notice that it follows from identity (3.2) that $\frac{1}{\bar{B}_{q}(x, i u)}$ is of the form $\mu_{1}[0, x]$, and we can use the identity $\left(\check{B}_{q}(x, i u)=\int_{] x,+\infty[} e^{-i u y} \check{C}_{q}(x, i u) \frac{H_{q}(d y)}{\breve{H}_{q}(y)}\right)$ of Theorem 4.1 in order to apply formula (10.3) to $\mu_{1}[0, x]:=\frac{1}{B_{q}(x, i u)}=\frac{1}{\left.\left.\mu_{2}\right] x,+\infty\right]}$ and get

$$
\left.\mu_{1}(d x)=\frac{\check{C}_{q}(x, i u)}{\check{B}_{q}\left(x^{-}, i u\right) \check{B}_{q}(x, i u)} e^{-i u x} \frac{H_{q}(d x)}{\check{H}_{q}(x)} \quad \text { on }\right] 0,+\infty[.
$$

In order to establish the equation relative to $\mu_{2}$, we first establish bounded variations on $[x,+\infty]$ of $C_{q}\left(x^{-}, i u\right)+(\phi(i u)+q) A_{q}\left(x^{-}, i u\right)$ as follows: the measures $\mathbf{Q}_{q}^{\uparrow}(d w)$ and $H_{q}(d x)$ are finite because $q$ is positive; thus the function $A_{q}\left(x^{-}, i u\right)=$ $\mathbf{Q}_{q}^{\uparrow}\left(M<x ; e^{-i u F}\right)$ tends to $\mathbf{Q}_{q}^{\uparrow}\left(e^{-i u F}\right)=\frac{1}{\psi_{q}(i u)}$ as $x$ goes to $+\infty$. On the other hand, the function of $x, \frac{\breve{A}_{q}(x, i u)}{\ddot{H}_{q}(x)}$, is bounded by 1 ; thus it is $H_{q}(d x)$ integrable. From these facts and from the identity of Theorem $4.1\left(A_{q}\left(x^{-}, i u\right)=\right.$ $\left.H_{q}(0)+\int_{] 0, x[} e^{-i u y} \frac{\check{A}_{q}(y, i u)}{\check{H}_{q}(y)} H_{q}(d y)\right)$, we deduce

$$
A_{q}\left(x^{-}, i u\right)=\frac{1}{\psi_{q}(i u)}-\int_{[x,+\infty[} e^{-i u y} \check{A}_{q}(y, i u) \frac{H_{q}(d y)}{\check{H}_{q}(y)} .
$$

Consequently, this equation and the identity of Theorem $4.1\left(C_{q}\left(x^{-}, i u\right)=\right.$ $\left.\int_{[x,+\infty[} e^{-i u y} B_{q}(x, i u) \frac{H_{q}(d y)}{H_{q}(y)}\right)$ give us that the function $C_{q}\left(x^{-}, i u\right)+(\phi(i u)+q)$ $A_{q}\left(x^{-}, i u\right)$ is of the form $\mu_{2}[x,+\infty]$ with

$$
\left.\mu_{2}(d x)=\left(B_{q}(x, i u)-(\phi(i u)+q) \check{A}_{q}(x, i u)\right) e^{-i u x} \frac{H_{q}(d x)}{\check{H}_{q}(x)} \quad \text { on }\right] 0,+\infty[.
$$

Now, we can integrate equation (10.7) over $] x,+\infty]$ and use equation (10.2) and obtain that the function

$$
\left.\left.\mu_{1}[0, x] \mu_{2}\right] x,+\infty\right]=\frac{C_{q}(x, i u)+(\phi(i u)+q) A_{q}(x, i u)}{\check{B}_{q}(x, i u)}
$$

is a constant function of $x$ on $] 0,+\infty[$. On the other hand,

$$
\lim _{x \rightarrow+\infty} C_{q}(x, i u)=0, \quad \lim _{x \rightarrow+\infty} A_{q}(x, i u)=\frac{1}{\psi_{q}(i u)}, \quad \lim _{x \rightarrow+\infty} \check{B}_{q}(x, i u)=\check{\psi}_{q}(i u) .
$$

Thus

$$
\lim _{x \rightarrow+\infty} \frac{C_{q}(x, i u)+(\phi(i u)+q) A_{q}(x, i u)}{\check{B}_{q}(x, i u)}=\frac{\phi(i u)+q}{\psi_{q}(i u) \check{\psi}_{q}(i u)}=1 .
$$


This enables us to deduce the second identity of Lemma 10.1

$$
\check{B}_{q}(x, i u)=C_{q}(x, i u)+(\phi(i u)+q) A_{q}(x, i u) .
$$

The third identity of Lemma 10.1 can be proved similarly or by regularizing the second one at the left and integrating it over $] x,+\infty]$ with respect to the measure $e^{i u x} \frac{\breve{H}_{q}(d x)}{H_{q}\left(x^{-}\right)}$. To get the second and the third identity when $q=0$, use a limit.

Enlarge the probability space $(\Omega, \mathcal{F}, \mathbf{P})$ so that it contains a random variable $\xi_{q}$ independent of the canonical process $X$ and which has an exponential distribution with parameter $q$ and still denote by the same notation this enlarged space. For $t>0$, write

$$
D_{t}:=\inf (] t,+\infty[\cap \operatorname{supp}(L)), \quad \check{D}_{t}:=\inf (] t,+\infty[\cap \operatorname{supp}(\check{L})) .
$$

Also set

$$
U^{x+}:=\inf \left\{t \mid S_{t}-I_{t}>x\right\}
$$

Lemma 10.2. For every complex number with $\Re(\lambda) \geq 0$,

$$
\begin{aligned}
& 1-\psi_{0}(\lambda) A_{q}(x, \lambda)=\mathbf{P}\left(\exp \left(-\lambda S_{D_{U^{x+\wedge}}}\right) ; D_{U^{x+\wedge}}<+\infty\right), \\
& 1-\frac{\psi_{0}(\lambda)}{B_{q}(x, \lambda)}=\mathbf{P}\left(\exp \left(-\lambda S_{D_{V_{x} \wedge \xi_{q}}}\right) ; D_{V_{x} \wedge \xi_{q}}<+\infty\right) .
\end{aligned}
$$

For every complex number with $\Re(\lambda) \leq 0$,

$$
\begin{aligned}
& 1-\check{\psi}_{0}(\lambda) \check{A}_{q}(x, \lambda)=\mathbf{P}\left(\exp \left(-\lambda I_{\check{D}_{U^{x}+\wedge \xi_{q}}}\right) ; \check{D}_{U^{x+\wedge \xi_{q}}}<+\infty\right), \\
& 1-\frac{\check{\psi}_{0}(\lambda)}{\check{B}_{q}(x, \lambda)}=\mathbf{P}\left(\exp \left(-\lambda I_{\check{D}_{V^{x} \wedge \xi_{q}}}\right) ; \check{D}_{V^{x} \wedge \xi_{q}}<+\infty\right) .
\end{aligned}
$$

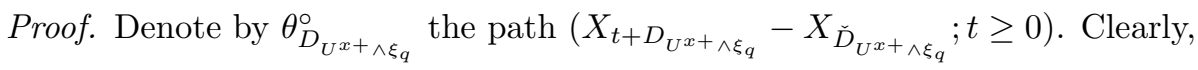

$$
\begin{aligned}
& \int_{[0, \infty[} e^{-\lambda S_{t}} L(d t)=\int_{\left[0, U^{x+} \wedge \xi_{q}\right]} e^{-\lambda S_{t}} L(d t)
\end{aligned}
$$

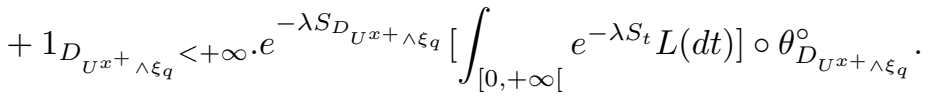

By using the Markov property at time $D_{U^{x+\wedge} \xi_{q}}$, we then obtain

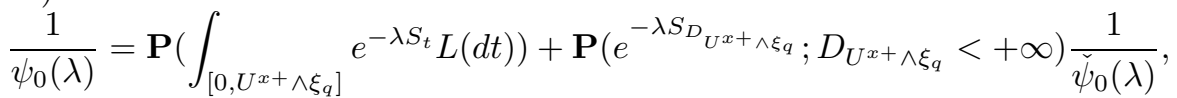

and we deduce from the definition of the function $A_{q}(x, \lambda)$ that

$$
A_{q}(x, \lambda)=\mathbf{P}\left(\int_{\left[0, U^{\left.x+\wedge \xi_{q}\right]}\right.} e^{-\lambda S_{t}} L(d t)\right) .
$$

The first identity of Lemma 10.2 follows from (10.8) and (10.9).

Use similar arguments to prove the other identities of the lemma. 
10.2. The matrix $M_{q}$ satisfies the properties of Theorem 4.2, Fact 1) is obvious. Fact 2) follows from the second and the third identities of Lemma 10.1 We check fact 3) :

The functions $\lambda \mapsto A_{q}(x, \lambda), \lambda \mapsto \check{A}_{q}(x, \lambda), \lambda \mapsto C_{q}(x, \lambda), \lambda \mapsto \check{C}_{q}(x, \lambda)$ are Laplace transforms of finite measures supported respectively by sets $[0, x],[-x, 0]$, $[x,+\infty[$ and $]-\infty, x]$. Consequently, the functions $\lambda \mapsto A_{q}(x, \lambda), \lambda \mapsto e^{-\lambda x} \check{A}_{q}(x, \lambda)$, $\lambda \mapsto e^{\lambda x} C_{q}(x, \lambda)$ are bounded on the half-plane $\{\Re(\lambda)>0\}$ and the functions $\lambda \mapsto e^{\lambda x} A_{q}(x, \lambda), \lambda \mapsto \check{A}_{q}(x, \lambda), \lambda \mapsto e^{-\lambda x} \check{C}_{q}(x, \lambda)$ are bounded on the half-plane $\{\Re(\lambda)<0\}$.

The function $\lambda \mapsto B_{q}(x, \lambda)$ (resp. $\lambda \mapsto \check{B}_{q}(x, \lambda)$ ) is the Lévy exponent of a subordinator (resp. opposite of a subordinator) killed at an exponential independent time. Thus the function $\lambda \rightarrow \frac{B_{q}(x, \lambda)}{|\lambda|+1}$ (resp. $\lambda \rightarrow \frac{\check{B}_{q}(x, \lambda)}{|\lambda|+1}$ ) is bounded on the half-plane $\{\Re(\lambda)>0\}$ (resp. $\{\Re(\lambda)<0\}$ ).

We now check fact 4$)$. Notice that the random variables $S_{D_{U^{x}+\wedge \xi_{q}}}$ and $S_{D_{V_{x} \wedge \xi_{q}}}$ (resp. $\quad I_{\check{D}_{U^{x+} \wedge \xi_{q}}}$ and $I_{\check{D}_{V^{x}} \wedge \xi_{q}}$ ) are positive (resp. negative). Thus by using Lemma 10.2, we obtain

$$
\begin{array}{ll}
\lim _{\Re(\lambda) \rightarrow+\infty} \psi_{0}(\lambda) A_{q}(x, \lambda)=1, & \lim _{\Re(\lambda) \rightarrow+\infty} \frac{B_{q}(x, \lambda)}{\psi_{0}(\lambda)}=1, \\
\lim _{\Re(\lambda) \rightarrow-\infty} \check{\psi}_{0}(\lambda) \check{A}_{q}(x, \lambda)=1, & \lim _{\Re(\lambda) \rightarrow-\infty} \frac{\check{B}_{q}(x, \lambda)}{\check{\psi}_{0}(\lambda)}=1 .
\end{array}
$$

Now, suppose $]-\infty, 0\left[\right.$ is regular and denote by $A_{q}(x, d y)$ the measure with Laplace transform $A_{q}(x, \lambda)$,

$$
A_{q}(x, d y):=\mathbf{P}\left(\int_{0}^{\zeta} 1_{S_{t}-I_{t} \leq x} e^{-q t} 1_{S_{t} \in d y} L(d t)\right) .
$$

In particular,

$$
A_{q}(x,\{x\})=\mathbf{P}\left(\int_{0}^{\zeta} 1_{I_{t}=0} e^{-q t} 1_{S_{t}=x} L(d t)\right) .
$$

Since ] $-\infty, 0\left[\right.$ is regular, $I_{t}=0$ if and only if $t=0$ and at time $t=0, S_{t}=S_{0}=$ $0 \neq x$. Hence, the measure $A_{q}(x, d y)$ does not change $\{x\}$ and $e^{\lambda x} A_{q}(x, \lambda)$ is a Laplace transform of a measure supported by $[-x, 0[$. Therefore,

$$
\lim _{\Re(\lambda) \rightarrow-\infty} e^{\lambda x} A_{q}(x, \lambda)=0 .
$$

When $] 0,+\infty[$ is regular, one similarly obtains the following limit:

$$
\lim _{\Re(\lambda) \rightarrow+\infty} e^{-\lambda x} \check{A}_{q}(x, \lambda)=0 .
$$

If ] $-\infty, 0$ [ is irregular, then for every positive stopping time $T$, either $\mathbf{P}$ is the distribution of a compound Poisson process and the set $\left\{s \mid \inf _{0 \leq u \leq s} X_{u+T}-X_{T^{-}}=\right.$ $\left.X_{s+T}-X_{T^{-}}\right\}$is (P-a.s.), a discrete union of intervals where the process $X$ is constant, or the set $\left\{s \mid \inf _{0 \leq u \leq s} X_{u+T}-X_{T^{-}}=X_{s+T}-X_{T^{-}}\right\}$is discrete. One can easily deduce that the set $\left\{t \mid I_{t}=X_{t}\right\}$ has the same property $\check{N}$-a.s. Moreover, the time $T_{x}$ is the first time when $X_{t}$ is strictly smaller than $-x$. It follows that $X_{T_{x}}<-x$ on $T_{x}<+\infty \check{N}$-a.s., and $\lambda \mapsto \check{C}_{q}(x, \lambda)=\check{N}\left(e^{-\lambda X_{T_{x}}-q T_{x}} ; T_{x}<+\infty\right)$ is the Laplace transform of a finite measure supported by $]-\infty,-x[$; thus

$$
\lim _{\Re(\lambda) \rightarrow-\infty} e^{-\lambda x} \check{C}_{q}(x, \lambda)=0 .
$$


When $] 0,+\infty[$ is irregular, then one similarly obtains the following limit:

$$
\lim _{\Re(\lambda) \rightarrow+\infty} e^{\lambda x} C_{q}(x, \lambda)=0 .
$$

10.3. Proof of the uniqueness part of Theorem 4.2. Notice first that, when regularizing on the right the identities $A_{q}\left(x^{-}, \lambda\right) B_{q}(x, \lambda)+\check{A}_{q}(x, \lambda) C_{q}\left(x^{-}, \lambda\right)=1$ and $\check{A}_{q}(x, \lambda) \check{B}_{q}\left(x^{-}, \lambda\right)+A_{q}\left(x^{-}, \lambda\right) \check{C}_{q}(x, \lambda)=1$ stated in Theorem 4.1] we obtain that $\operatorname{det} M_{q}(x, \lambda)=1$ for every $\lambda \in \mathbf{C} \backslash i \mathbf{R}$. Now let $N(\lambda)$ be another matrix having the same property as $M_{q}(x, \lambda)$ and let us check that $N(\lambda)\left[M_{q}(x, \lambda)\right]^{-1}$ is equal to the identity matrix. For simplicity, the mention of parameters $x$ and $q$ is omitted in the sequel.

By fact 1) the matrix $N(\lambda)[M(\lambda)]^{-1}$ is analytic on the two half-planes $\{\Re(\lambda)>$ $0\}$ and $\{\Re(\lambda)<0\}$, and by fact 2$)$ it can be extended by continuity at every point of the imaginary axis.

Fact 3) allows us to state that $N(\lambda) M(\lambda)^{-1}$ is bounded on every compact set of C. Thus a standard argument shows that the extended matrix is entire.

Fact 3) gives us also that the matrix $\frac{1}{(|\lambda|+1)^{2}} e^{\lambda \frac{x}{2} J} N(\lambda)[M(\lambda)]^{-1} e^{-\lambda \frac{x}{2} J}$ is bounded on $\mathbf{C}$ (remember that $J$ denotes the matrix $\left(\begin{array}{ll}1 & 0 \\ 0 & -1\end{array}\right)$ ); hence the components of the matrix $U:=e^{\lambda \frac{x}{2} J} N(\lambda)[M(\lambda)]^{-1} e^{-\lambda \frac{x}{2} J}$ are polynomials.

We now investigate the behavior of each component of this matrix as $\Re(\lambda)$ goes to $-\infty$ or $+\infty$. Let us compute $U_{11}$,

$$
U_{11}=N_{11} M_{22}-N_{12} M_{21} .
$$

If ] $-\infty, 0$ [ is regular, then $e^{\lambda x} N_{12}$ goes to 0 as $\Re(\lambda)$ goes to $-\infty$, while $e^{-\lambda x} M_{21}$ is bounded; therefore, the product $N_{12} M_{21}$ goes to 0 . On the other hand, $N_{11} \sim \breve{\psi}(\lambda)$ and $M_{22} \sim \frac{1}{\psi(\lambda)}$; thus $N_{11} M_{22} \rightarrow 1$. We have obtained that $U_{11}$ goes to 1 as $\Re(\lambda)$ goes to $-\infty$ and $U_{11}$ is a polynomial; thus $U_{11}$ is identically 1 .

If $] 0,+\infty$ [ is regular, exchange the roles of the two half-planes in order to obtain that $U_{11}$ is equal to 1 again.

In other cases, $\mathbf{P}$ is the distribution of a compound Poisson process, and so $e^{-\lambda x} M_{21}$ goes to 0 as $\Re(\lambda)$ goes to $-\infty$, while $e^{\lambda x} N_{12}$ is bounded; thus the product $N_{12} M_{21}$ goes to 0 again. Moreover, $N_{11} M_{22}$ goes to 1 as before. We can again conclude that $U_{11}$ goes to 1 as $\Re(\lambda)$ goes to $-\infty$, and so, that $U_{11}$ is identically 1 .

We turn now to the computation of $U_{22}$,

$$
U_{22}=N_{22} M_{11}-N_{21} M_{12} .
$$

Upon exchanging the roles of $N$ and $M$ in the previous argument, we get that $U_{22}$ is identically 1 .

Compute $U_{21}$,

$$
U_{21}=e^{-\lambda x}\left(N_{21} M_{22}-N_{22} M_{21}\right) .
$$

If $]-\infty, 0$ [ is regular, then $\check{\psi}_{0}$ is the exponent of the opposite of a subordinator that is not a compound Poisson process, and $M_{22}$ and $N_{22}$ are equivalent to $\frac{1}{\psi_{0}(\lambda)}$; thus they vanish when $\Re(\lambda)$ goes to $-\infty$. Moreover, $e^{-\lambda x} N_{21}$ and $e^{-\lambda x} M_{21}$ are bounded, so $U_{21}$ vanishes as $\Re(\lambda)$ goes to $-\infty$ and we conclude that $U_{21}$ is identically 0 .

If $]-\infty, 0\left[\right.$ is irregular, then $\check{\psi}_{0}$ is the exponent of the opposite of a subordinator which is a compound Poisson process. Then it converges to a positive value as $\Re(\lambda)$ 
goes to $-\infty$. Thus, $\lim _{\Re(\lambda) \rightarrow-\infty} M_{22}=\lim _{\Re(\lambda) \rightarrow-\infty} N_{22}=\lim _{\Re(\lambda) \rightarrow-\infty} \frac{1}{\dot{\psi}(\lambda)} \in$ ]0, $+\infty$ [. On the other hand, $e^{-\lambda x} N_{21}$ and $e^{-\lambda x} M_{21}$ go to 0 . Therefore, $U_{21}$ goes to 0 as $\Re(\lambda)$ goes to $-\infty$ and again $U_{21}$ is identically 0 .

Finally, compute $U_{12}$,

$$
U_{12}=e^{\lambda x}\left(-N_{11} M_{21}+N_{21} M_{11}\right)
$$

By a similar argument, we obtain that $U_{12}$ goes to 0 as $\Re(\lambda)$ goes to $+\infty$ and again $U_{12}$ is identically 0 .

We have obtained that the matrix $U=e^{\lambda \frac{x}{2} J} N(\lambda)[M(\lambda)]^{-1} e^{-\lambda \frac{x}{2} J}$ is equal to the identity matrix; thus $N(\lambda)=M(\lambda)$.

\section{Probabilistic interpretation of the identities BETWEEN THE SIX FUNCTIONS}

We first introduce some terminology on Wiener-Hopf factorization. Denote by $(W, \mathcal{G}, \mathbf{Q})$ any probability space. Let $Z$ be a real process defined on $(W, \mathcal{G})$ with a lifetime that may be finite. If the distribution of $Z$ under $\mathbf{Q}$ is $\mathbf{P}_{q}$, we shall say that $\phi(i u)+q$ is the Lévy exponent of $Z$ under $\mathbf{Q}$ and its spatial Wiener-Hopf factors are the functions $\check{\psi}_{q}$ and $\psi_{q}$.

Let $S=\left(0, S_{1}, \ldots, S_{n}, \ldots\right)$ be a sequence of real random variables defined on $(W, \mathcal{G})$ and having under $\mathbf{Q}$ the distribution of a random walk possibly killed at an independent geometric time. We will identify its distribution to the distribution of the compound Poisson process with Lévy exponent $1-\mathbf{Q}\left(e^{-i u S_{1}} ; 1<\xi\right)(\xi$ denotes the lifetime of $\left.S, \xi=\inf \left\{n \mid S_{n}=\delta\right\}\right)$. We shall say that this function is the Lévy exponent of the random walk $S$ and that its spatial Wiener-Hopf factors are those of this compound Poisson process. It is easily seen that these factors are respectively $1-\mathbf{Q}\left(e^{-i u S_{V_{0}}} ; V_{0}<\xi\right)$ and $1-\mathbf{Q}\left(e^{-i u S_{V^{0}}} ; V^{0-}<\xi\right) .\left(V_{0}:=\inf \left\{n \geq 1 \mid S_{n}<0\right\}\right)$ and $\left.V^{0-}:=\inf \left\{n \geq 1 \mid S_{n} \geq 0\right\}\right)$.

We now revert to the space $(\Omega, \mathcal{F}, \mathbf{P})$ and denote by $\theta_{t}^{\circ}$ the path $\left(X_{s+t}-X_{t} ; s \geq 0\right)$ for every time $t$. The reader can easily convince him/herself of the next assertions:

11.1. Interpretation of the identity $\check{B}_{q}(x, i u)=(\phi(i u)+q) A_{q}(x, i u)+C_{q}(x, i u)$. If $]-\infty, 0[$ is regular, then define:

$$
\begin{aligned}
T_{0} & :=0, \quad T_{1}:=V^{x}, \quad T_{n+1}:=T_{n}+V^{x} \circ \theta_{T_{n}}^{\circ}, \quad\left(T_{n+1}:=+\infty \text { if } T_{n}=+\infty\right), \\
\tilde{L}(d t) & :=\sum_{n=0}^{+\infty} 1_{T_{n} \leq t<T_{n+1}} \check{L}\left(\theta_{T_{n}}^{\circ}, d t-T_{n}\right) .
\end{aligned}
$$

Denote by $\left(\tilde{\tau}_{t}\right)$ the right continuous inverse of $\tilde{\tilde{L}}$ and write

$$
Y_{t}:=X_{\tilde{\tau}_{t}}
$$

Under $\mathbf{P}_{q}, Y$ is a Lévy process, its Lévy exponent is $\check{B}_{q}(x, i u)-C_{q}(x, i u)$ and its Wiener-Hopf spatial factors are $\check{\psi}_{q}(i u)$ and $\psi_{q}(i u) A_{q}(x, i u)$.

If ] $-\infty, 0$ [ is irregular, then define the sequence of stopping times $\left(T_{n} ; n \geq 1\right)$ as follows:

$T_{1}:=T_{0}^{x}=\inf \left\{t \mid X_{t} \notin[0, x]\right\}, T_{n+1}:=T_{n}+T_{0}^{x} \circ \theta_{T_{n}}^{\circ},\left(T_{n+1}:=+\infty\right.$ if $\left.T_{n}=+\infty\right)$.

Under $\mathbf{P}_{q}$, the sequence $\left(0, X_{T_{1}}, \ldots, X_{T_{n}}, \ldots\right)$ is a random walk. Its Lévy exponent is $\check{B}_{q}(x, i u)-C_{q}(x, i u)$ and its Wiener-Hopf spatial factors are $\check{\psi}_{q}(i u)$ and $\psi_{q}(i u) A_{q}(x, i u)$. 
The interpretation of the identity $B_{q}(x, i u)=(\phi(i u)+q) \check{A}_{q}(x, i u)+\check{C}_{q}(x, i u)$ is similar.

\subsection{Interpretation of the identity.}

$$
(\phi(i u)+q) \check{A}_{q}(x, i u) A_{q}\left(x^{-}, i u\right)+C_{q}\left(x^{-}, i u\right) \check{A}_{q}(x, i u)+\check{C}_{q}(x, i u) A_{q}\left(x^{-}, i u\right)=1 .
$$

This identity is obtained from

$$
B_{q}(x, i u) A_{q}\left(x^{-}, i u\right)+C_{q}\left(x^{-}, i u\right) \check{A}_{q}(x, i u)=1
$$

and

$$
B_{q}(x, i u)=(\phi(i u)+q) \check{A}_{q}(x, i u)+\check{C}_{q}(x, i u) .
$$

Denote by $\left(U_{n} ; n \geq 1\right)$ the sequence of stopping times :

$$
U_{1}:=U^{x}, \quad U_{n+1}:=U_{n}+U^{x} \circ \theta_{U_{n}}^{\circ} \quad\left(U_{n+1}:=+\infty \text { if } U_{n}=+\infty\right) .
$$

Under $\mathbf{P}_{q}$, the sequence $\left(0, X_{U_{1}}, \ldots, X_{U_{n}}, \ldots\right)$ is a random walk, its Lévy exponent is $1-C_{q}\left(x^{-}, i u\right) \check{A}_{q}(x, i u)-\check{C}_{q}(x, i u) A_{q}\left(x^{-}, i u\right)$ and its Wiener-Hopf spatial factors are $\check{\psi}_{q}(i u) \check{A}_{q}(x, i u)$ and $\psi_{q}(i u) A_{q}\left(x^{-}, i u\right)$.

11.3. Interpretation of the identity $\check{B}_{q}\left(x^{-}, i u\right) B_{q}(x, i u)-C_{q}\left(x^{-}, i u\right) \check{C}_{q}(x, i u)=$ $\phi(i u)+q$.

Let $S=\left(0, S_{1}, \ldots, S_{n}, \ldots\right)$ be a real-valued random walk (with infinite liftime) on any probability space $(W, \mathcal{G}, \mathbf{Q})$. Denote by, for all $x \in] 0,+\infty[$,

$$
V_{0}^{x-}=\inf \left\{n \geq 1 \mid S_{n} \notin\left[0, x[\}, \quad V_{x}^{0-}=\inf \left\{n \geq 1 \mid S_{n} \notin[-x, 0[\} .\right.\right.\right.
$$

When applying the identity : $\check{B}_{q}\left(x^{-}, i u\right) B_{q}(x, i u)-\check{C}_{q}(x, i u) C_{q}\left(x^{-}, i u\right)=\phi(i u)+q$ to the compound Poisson process which has Lévy exponent $1-\mathbf{Q}\left(e^{-i u S_{1}}\right)=\phi(i u)$, we obtain for all $i u \in i \mathbf{R}$ and $s \in[0,1]$,

$$
\begin{aligned}
& {\left[1-\mathbf{Q}\left(e^{-i u S_{V_{0}^{x-}} s^{x-}} ; S_{V_{0}^{x-}}<0\right)\right]\left[1-\mathbf{Q}\left(e^{-i u S_{V_{x}^{0-}}} s^{V_{x}^{0-}} ; S_{V_{x}^{0-}} \geq 0\right)\right]} \\
& -\mathbf{Q}\left(e^{-i u S_{V_{0}^{x-}} s^{x-}} ; S_{V_{0}^{x-}} \geq x\right) \mathbf{Q}\left(e^{-i u S_{V_{x}^{0-}} s^{V_{x}^{0-}}} ; S_{V_{x}^{0-}}<-x\right)=1-s \mathbf{Q}\left(e^{-i u S_{1}}\right) \text {. }
\end{aligned}
$$

\section{EXAmples}

In this section, we treat two examples of Lévy processes: stable processes which are not killed $[q=0]$ and Lévy processes without positive jumps. The "exit problem" has been essentially solved for these processes, by Rogozin [19] in the first case and by Takács 24] in the second case. The authors mentioned in part 3 gave solutions of derived problems. This section is meant to show how the main results presented here (Theorems 4.1 and 4.2) can be used to provide a unified treatment of the subject. The reader can also find in the recent work of [11] other cases for which the "exit problem" is solved.

12.1. Stable processes. Let $\mathbf{P}$ be the distribution of the stable process with index $\alpha$, with positivity parameter $\rho$ (i.e., $\rho=\mathbf{P}\left(X_{1}>0\right)$ ), and normalized by the equation $|\phi(i)|=1$. Put $\gamma:=\alpha \rho$ and $\delta:=\alpha(1-\rho)$, where $\gamma$ and $\delta$ belong to the interval $[0,1]$. The case $\delta=0$ (resp. $\gamma=0$ ) corresponds to the subordinators (resp. the opposite of subordinators) and we will exclude these cases in the sequel. The case $\gamma=1$ corresponds to stable processes without positive jumps. The Lévy exponent $\phi$ can be written as follows (we use the principal part of the power functions):

$$
\phi(i u)=e^{i \frac{\pi}{2}(\gamma-\delta) \epsilon(u)}|u|^{\alpha}=(-i u)^{\delta}(i u)^{\gamma} .
$$


We chose the spatial Wiener-Hopf factors

$$
\check{\psi}_{0}(i u)=(-i u)^{\delta}, \quad \psi_{0}(i u)=(i u)^{\gamma} .
$$

Theorem 12.1. For all $x \in] 0,+\infty[$,

$$
A_{0}(x, \lambda)=\frac{1}{\Gamma(\gamma)} \int_{0}^{x} e^{-\lambda y} y^{\gamma-1}\left(1-\frac{y}{x}\right)^{\delta} d y \quad(\lambda \in \mathbf{C})
$$

for $\gamma \in] 0,1[$,

$$
\begin{aligned}
& C_{0}(x, \lambda)=\frac{\Gamma(\delta+1)}{\Gamma(1-\gamma) \Gamma(\gamma)} \int_{x}^{+\infty} e^{-\lambda y}\left(\frac{y}{x}-1\right)^{-\gamma} y^{-\delta-1} d y \quad(\Re(\lambda) \geq 0), \\
& B_{0}(x, \lambda)=\frac{\gamma}{\Gamma(1-\gamma)} \int_{0}^{+\infty}\left(1-e^{-\lambda y}\right)\left(\frac{y}{x}+1\right)^{\delta} y^{-\gamma-1} d y+\frac{\Gamma(\alpha)}{\Gamma(\delta)} x^{-\gamma} \quad(\Re(\lambda) \geq 0)
\end{aligned}
$$

and for $\gamma=1$ (consequently $\delta=\alpha-1$ ),

$$
\begin{aligned}
& C_{0}(x, \lambda)=\Gamma(\alpha) \cdot e^{-\lambda x} x^{-\delta} \quad(\Re(\lambda) \geq 0), \\
& B_{0}(x, \lambda)=\lambda+\frac{\delta}{x} \quad(\Re(\lambda) \geq 0) .
\end{aligned}
$$

The functions $\check{A}_{0}, \check{C}_{0}$ and $\check{B}_{0}$ are obtained by duality.

Sketch of proof. The stability property gives us easily that the functions $H_{0}$ and $\check{H}_{0}$ are of the form

$$
H_{0}(x)=k_{+} x^{\gamma}, \quad \check{H}_{0}(x)=k_{-} x^{\delta},
$$

where $k_{+}$and $k_{-}$are positive constants. Consequently, upon differentiating twice the integral equation of Theorem 4.1. one finds that the differential equation in $x$, satisfied by the three functions $A_{0}(x, \lambda), C_{0}(x, \lambda)$ and $\check{B}_{0}(x, \lambda)$, is the confluent hypergeometric equation. This fact and the behavior of these functions when $x$ goes to $+\infty$ allows us to compute them.

12.2. Lévy processes without positive jumps. In this section $\mathbf{P}$ is the distribution of a Lévy process without positive jumps. We refer to chapter 7 of the book [3] for the results we recall here. The Lévy exponent is of the form

$$
\phi(i u)=\sigma^{2} u^{2}+a i u+\int_{]-\infty, 0[}\left(1-e^{-i u x}-i u x 1_{x>-1}\right) \pi(d x) .
$$

This function can be extended to define an analytic function on the open half-plane $\Re(\lambda)<0\}$ which is continuous on the closed half-plane $\{\Re(\lambda) \leq 0\}$. Again, let $\phi$ be this function. Moreover, there exists a unique function $\Psi$ defined on $[0,+\infty[$ such that $\phi(-\Psi(q))=-q$, and one can use as Wiener-Hopf factor $\psi_{q}$ the function

$$
\psi_{q}(\lambda)=\lambda+\Psi(q)
$$

This Wiener-Hopf factor corresponds to the choice of the local time $L(d t)=d S_{t}$. Notice that in case $\mathbf{P}$ is the law of a Lévy process of the form at $-Y_{t}$, where $a$ is a positive real and $Y$ is a subordinator without drift, one has to take $\check{L}(d t)=$ $\frac{1}{a} \sum 1_{I_{t}=X_{t}} \delta_{t}$ instead of $\check{L}(d t)=\sum 1_{I_{t}=X_{t}} \delta_{t}$ as we have stated at the beginning of this paper but this doesn't change previous results. Denote by $W_{q}$ the so-called scale function. The scale function is the unique nondecreasing right continuous function defined for $q \in[0,+\infty[$ and for $x \in[0,+\infty[$ and satisfying the identity

$$
\int_{0}^{+\infty} e^{-\lambda x} W_{q}(x) d x=\frac{1}{-\phi(-\lambda)-q} \quad(\lambda>\Psi(q)) .
$$


The associated Stieljes measure $W_{q}(d x)$ admits a right continuous density $w_{q}$ on ] $0,+\infty[$ (we shall reprove this fact in the next proof)

$$
W_{q}(x)=W_{q}(0)+\int_{] 0, x]} w_{q}(y) d y .
$$

Theorem 12.2. For all $q \in[0,+\infty[$ and $x \in] 0,+\infty[$,

$$
\begin{aligned}
& A_{q}(x, \lambda)=\int_{0}^{x} e^{-\lambda y} \frac{W_{q}(x-y)}{W_{q}(x)} d y \quad(\lambda \in \mathbf{C}), \\
& \check{A}_{q}(x, \lambda)=W_{q}(0)+\int_{-x}^{0} e^{-\lambda y}\left[w_{q}(-y)-\frac{w_{q}(x)}{W_{q}(x)} W_{q}(-y)\right] d y \quad(\lambda \in \mathbf{C}) .
\end{aligned}
$$

The functions $C_{q}$ and $B_{q}$ can be analytically extended to the whole complex plane and

$$
\begin{array}{rlrl}
C_{q}(x, \lambda) & =\frac{e^{-\lambda x}}{W_{q}(x)} \quad(\lambda \in \mathbf{C}), \\
B_{q}(x, \lambda) & =\lambda+\frac{w_{q}(x)}{W_{q}(x)} \quad(\lambda \in \mathbf{C}), \\
\check{C}_{q}(x, \lambda) & =B_{q}(x, \lambda)-(\phi(\lambda)+q) \check{A}_{q}(x, \lambda) & & (\Re(\lambda) \leq 0), \\
\check{B}_{q}(x, \lambda) & =(\phi(\lambda)+q) A_{q}(x, \lambda)+C_{q}(x, \lambda) & & (\Re(\lambda) \leq 0) .
\end{array}
$$

Proof. Since there are no positive jumps, we see that

$$
C_{q}\left(x^{-}, \lambda\right)=e^{-\lambda x} N\left(S_{\xi_{q}} \geq x\right),
$$

$$
B_{q}(x, \lambda)=\lambda+\check{N}\left(\xi_{q}<\zeta \text { or } I_{\xi_{q}}<-x\right) \text { and } \lim _{x \rightarrow+\infty} \check{N}\left(\xi_{q}<\zeta \text { or } I_{\xi_{q}}<-x\right)=\Psi(q) \text {, }
$$

where $\xi_{q}$ denotes an independent exponential time after having enlarged the measures $N$ and $\Sigma$ in order to contain such a variable.

Differentiate the integral equation of Theorem 4.1

$$
C_{q}\left(x^{-}, \lambda\right)=\int_{[x,+\infty[} e^{-\lambda y} B_{q}(y, \lambda) \frac{H_{q}(d y)}{\check{H}_{q}(y)},
$$

and use the expressions of $C_{q}$ and $B_{q}$ given by (12.2) and (12.3) to deduce

$$
\left.\frac{H_{q}(d x)}{\check{H}_{q}(x)}=N\left(S_{\xi_{q}} \geq x\right) d x \quad \text { on }\right] 0,+\infty[,
$$

and the measure $N\left(S_{\xi_{q}} \in d x\right)$ admits a right continuous density $n_{q}$ on $] 0,+\infty[$ and

$$
\check{N}\left(\xi_{q}<\zeta \text { or } I_{\xi_{q}}<-x\right)=\frac{n_{q}(x)}{N\left(S_{\xi_{q}}>x\right)} \quad(x \in] 0,+\infty[) .
$$

In particular, we see that

$$
\lim _{x \rightarrow+\infty} \frac{n_{q}(x)}{N\left(S_{\xi_{q}}>x\right)}=\Psi(q) .
$$


Recall the following identity from Theorem 4.1;

$$
A_{q}\left(x^{-}, \lambda\right)=H_{q}(0)+\int_{] 0, x]} e^{-\lambda y} \check{A}_{q}(y, \lambda) \frac{H_{q}(d y)}{\check{H}_{q}(y)} .
$$

In view of equation (12.5), it follows from equations (12.4) and (12.7) that $C_{q}(x, \lambda)$ and $A_{q}(x, \lambda)$ are differenciable functions of $x$. Upon computing the value of $\left[\frac{A_{q}(x, \lambda)}{C_{q}(x, \lambda)}\right]^{\prime}$, we obtain

$$
\left[\frac{A_{q}(x, \lambda)}{C_{q}(x, \lambda)}\right]^{\prime}=\frac{B_{q}(x, \lambda) A_{q}(x, \lambda)+C_{q}(x, \lambda) \check{A}_{q}(x, \lambda)}{\left(C_{q}(x, \lambda)\right)^{2}} \frac{e^{-\lambda x} H_{q}(d x)}{\check{H}_{q}(x)}=e^{\lambda x} \frac{1}{N\left(S_{\xi_{q}}>x\right)} .
$$

The first identity is a consequence of identities (12.4) and (12.7), and the second one follows from (12.2) and (12.5) and from the identity of Theorem 4.1. $B_{q}(x, \lambda) A_{q}\left(x^{-}, \lambda\right)+C_{q}\left(x^{-}, \lambda\right) \check{A}_{q}(x, \lambda)=1$.

Upon integrating equation (12.8), we obtain

$$
A(x, \lambda)=C(x, \lambda) \int_{0}^{x} e^{\lambda y} \frac{1}{N\left(S_{\xi_{q}}>y\right)} d y=\int_{0}^{x} e^{-\lambda z} \frac{N\left(S_{\xi_{q}}>x\right)}{N\left(S_{\xi_{q}}>x-z\right)} d y .
$$

The second identity follows from (12.2).

In order to identify the function $\breve{A}_{q}(x, \lambda)$, differentiate the preceding one and use (12.7) to obtain

$$
\check{A}_{q}(x, \lambda)=\frac{1}{N\left(S_{\xi_{q}}>0\right)}+\int_{0}^{x} e^{\lambda y}\left(\frac{n_{q}(y)}{\left[N\left(S_{\xi_{q}}>y\right)\right]^{2}}-\frac{1}{N\left(S_{\xi_{q}}>y\right)} \frac{n_{q}(x)}{N\left(S_{\xi_{q}}>x\right)}\right) d y .
$$

We now prove that the function $\frac{1}{N\left(S_{\xi_{q}}>x\right)}$ is equal to the scale function $W_{q}(x)$, and this will complete the proof.

For $\Re(\lambda)<0$ and $x \rightarrow+\infty$, use (12.6) in equation (12.9) to obtain

$$
\lim _{x \rightarrow+\infty} \check{A}_{q}(x, \lambda)=\frac{1}{N\left(S_{\xi_{q}}>0\right)}+\int_{0}^{+\infty} e^{\lambda y}\left(\frac{n_{q}(y)}{\left[N\left(S_{\xi_{q}}>y\right)\right]^{2}}-\frac{\Psi(q)}{N\left(S_{\xi_{q}}>y\right)}\right) d y .
$$

Moreover, $\lim _{x \rightarrow+\infty} \check{A}_{q}(x, \lambda)=\frac{1}{\check{\psi}_{q}(\lambda)}=\frac{\lambda+\Psi(q)}{\phi(\lambda)+q}$. Therefore for $\Re(\lambda)<0$,

$$
\begin{aligned}
& \frac{\lambda+\Psi(q)}{\phi(\lambda)+q}=\frac{1}{N\left(S_{\xi_{q}}>0\right)}+\int_{0}^{+\infty} e^{\lambda y}\left(\frac{n_{q}(y)}{\left[N\left(S_{\xi_{q}}>y\right)\right]^{2}}-\frac{\Psi(q)}{N\left(S_{\xi_{q}}>y\right)}\right) d y \\
& \quad=\frac{1}{N\left(S_{\xi_{q}}>0\right)}+\int_{0}^{+\infty} e^{(\lambda+\Psi(q)) y}\left[\frac{1}{N\left(S_{\xi_{q}}>y\right)} e^{-\Psi(q) y]^{\prime} d y}\right. \\
& \quad=-(\lambda+\Psi(q)) \cdot \int_{0}^{+\infty} \frac{e^{\lambda y}}{N\left(S_{\xi_{q}}>y\right)} d y .
\end{aligned}
$$

The last identity follows from an integration by parts.

We deduce the following identity for $\Re(\lambda)<0$,

$$
-\frac{1}{\phi(\lambda)+q}=\int_{0}^{+\infty} e^{\lambda y} \frac{1}{N\left(S_{\xi_{q}}>y\right)} d y .
$$

Comparing with identity (12.1), one finds that the Laplace transforms of the two right continuous (in fact continuous) functions $\frac{1}{N\left(S_{\xi_{q}}>x\right)}$ and $W_{q}(x)$ are equal on $\{\Re(\lambda)>\Psi(q)\}$. Thus, these two functions are equal. 


\section{ACKNOWLEDGMENTS}

The author would like to thank the anonymous referee for the numerous improvements he has suggested, especially for pointing out many references.

\section{REFERENCES}

1. Avram, F., Kyprianou, A.E., Pistorius, M.R. Exit problems for spectrally negative Lévy processes and applications to (Canadized) Russian options. Ann. Appl. Probab. 14, 215-238 (2004). MR2023021 (2005c:60053)

2. Bertoin, J. Exponential decay and geometric ergodicity of completely asymmetric Lévy processes in a finite interval. Ann. Appl. Probab. 7, 156-167 (1997). MR1428754 (98d:60147)

3. Bertoin, J. Lévy processes. Cambridge University Press (1996). MR.1406564 (98e:60117)

4. Bingham, N.H. Fluctuation theory in continuous time. Adv. Appl. Probab. 7, 705-766, (1975). MR0386027 (52:6886)

5. Beals, R., Deift, P., Zhou, X. The inverse scattering transform on the line. Important Developments in Soliton Theory. A.S. Fokas, V.E. Zakharov (Eds). Springer-Verlag 7-33.(1993) MR.1280467 (95k:34020)

6. Doney, R. A. Some excursion calculations for spectrally one-sided Lévy processes. Sem. de Probab. XXXVIII, Lecture Notes in Math. 1857, 5-15, (2004). MR2126963 (2006b:60098)

7. Emery, D. J. Exit problem for a spectrally positive process. Adv. Appl. Probab. 5, 498-520, (1973). MR0341623 (49:6370)

8. Fourati, S. Points de croissance des processus de Lévy et théorie générale des processus. Probab. Theory Relat. Fields 110 13-49, (1998). MR.1602032 (99e:60164)

9. Fourati, S. Krein theory on strings applied to fluctuations of Lévy processes. Preprint. ArXiv: Math.PR/0508612v1 (2005).

10. Fourati, S. Fluctuation des processus de Lévy et dispersion ("scattering").C.R. Acad. Sci. 1342, 135-139, (2006). MR2193661

11. Karandov, V.F. and Karandova, T.V. On the distribution of the time of the first exit from an interval and value of a jump over the boundary for processes with independent increments and random walks. Ukainian Mathematical Journal Vol. 47, No. 10 (2005).

12. Koryluk, V.S., Suprun, V.N. and Shurenkov, V. M. Method of potential in boundary problems for processes with increases and jumps of the same sign. Theory. Probab. Appl. 21, 243-249(1976).

13. Kyprianou, A. E. First passage of reflected strictly stable processes ALEA 2, 119-123, (2006). MR2249665 (2008e:60139)

14. Kyprianou, A. E. and Palmowski, Z. A martingale review of some fluctuation theory for spectrally negative Lévy processes. Sem. de Probab. XXXVIII, Lecture Notes in Math. 1857, 16-29, (2004). MR2126964 (2005m:60103)

15. Nguyen-Ngoc, L. and Yor, M. Some martingales associated to reflected Lévy processes. Sem. de Probab. XXXVIII, Lecture Notes in Math., no. 1857, 42-69, (2005). MR2126966 (2006a:60081)

16. Pistorius, M. R. On exit and ergodicity of the spectrally negative Lévy process at its infimum. J. Theor. Probab. 17, 183-220, (2004). MR2054585(2005e:60104)

17. Pistorius, M. R. A potential theoretical review of some exit problems of spectrally negative Lévy processes. Sem. de Probab. XXXVIII, Lecture Notes in Math 1857, 42-69, (2004). MR 2126965 (2006k:60086)

18. Rogers, L. C. G. The two-sided exit problem for spectrally positive Lévy processes. Adv. Appl. Probab. 22, 486-487, (1990). MR 1053243 (93f:60110)

19. Rogozin, B. A. The distribution of the position of absorption for stable and asymptotically stable random walks on an interval. Theor. Probab. Appl. 17 (1972), 332-338. MR0300349 (45:9395)

20. Sato, K. Lévy processes and infinitely divisible distributions. Cambridge University Press (1999). MR.1739520 (2003b:60064)

21. Shabat, A. B. An inverse scattering problem. Differential Equations 15 1299-1307, (1980). MR.553630 (81m:34026)

22. Spitzer, F. A combinatorial lemma and its applications to probability theory. Trans. Amer. Math. Soc. 82 323-339, (1956). MR0079851(18:156e) 
23. Suprun, V. N. The ruin problem and the resolvent of a killed independent increment process. Ukrainian Math. J. 28, 39-45, (1976). MR0428476 (55:1497)

24. Takács, L. Combinatorial methods in the theory of stochastic processes. Wiley, New York (1967. ). MR0217858(36:947)

Laboratoire de Probabilities, University of Paris Vi, 4 Place Jussieu Tour 56, 75252 Paris Cedex 5, France

Current address: Place Emile Blondel 76131 Mont Saint Aignan, France

E-mail address: sonia.fourati@upmc.fr 